Accepted for publication in ApJ

Preprint typeset using $\mathrm{LAT}_{\mathrm{E}} \mathrm{X}$ style emulateapj v. 12/16/11

\title{
BARYONS MATTER: WHY LUMINOUS SATELLITE GALAXIES HAVE REDUCED CENTRAL MASSES
}

\author{
Adi Zolotov ${ }^{1}$, Alyson M. Brooks ${ }^{2}$, Beth Willman ${ }^{3}$, Fabio Governato ${ }^{4}$, Andrew Pontzen ${ }^{5}$, Charlotte \\ Christensen $^{6}$, Avishai Dekel ${ }^{1}$, Tom Quinn ${ }^{4}$, Sijing Shen $^{7}$, James Wadsley $^{8}$
}

(Dated: November 1, 2018)

Accepted for publication in ApJ

\section{ABSTRACT}

Using high resolution cosmological hydrodynamical simulations of Milky Way-massed disk galaxies, we demonstrate that supernovae feedback and tidal stripping lower the central masses of bright $\left(-15<M_{V}<-8\right)$ satellite galaxies. These simulations resolve high density regions, comparable to giant molecular clouds, where stars form. This resolution allows us to adopt a prescription for $\mathrm{H}_{2}$ formation and destruction that ties star formation to the presence of shielded, molecular gas. Before infall, supernova feedback from the clumpy, bursty star formation captured by this physically motivated model leads to reduced dark matter $(\mathrm{DM})$ densities and shallower inner density profiles in the massive satellite progenitors $\left(\mathrm{M}_{v i r} \geq 10^{9} M_{\odot}, \mathrm{M}_{*} \geq 10^{7} M_{\odot}\right)$ compared to DM-only simulations. The progenitors of the lower mass satellites are unable to maintain bursty star formation histories, due to both heating at reionization and gas loss from initial star forming events, preserving the steep inner density profile predicted by DM-only simulations. After infall, gas stripping from satellites reduces the total central masses of SPH satellites relative to DM-only satellites. Additionally, enhanced tidal stripping after infall due to the baryonic disk acts to further reduce the central DM densities of the luminous satellites. Satellites that enter with cored DM halos are particularly vulnerable to the tidal effects of the disk, exacerbating the discrepancy in the central masses predicted by baryon+DM and DM-only simulations. We show that DM-only simulations, which neglect the highly non-adiabatic evolution of baryons described in this work, produce denser satellites with larger central velocities. We provide a simple correction to the central DM mass predicted for satellites by DM-only simulations. We conclude that DM-only simulations should be used with great caution when interpreting kinematic observations of the Milky Way's dwarf satellites.

Subject headings: Galaxy — halo; galaxies — dwarfs

\section{INTRODUCTION}

The favored Cold Dark Matter (CDM) cosmological model has been successful in reproducing many large scale observable properties of the Universe (e.g., Efstathiou et al. 1992; Riess et al. 1998; Spergel et al. 2007). The CDM model, however, still faces many challenges from observations of galaxies on small scales. The most well known of these problems has been termed the "missing satellite problem", since CDMbased models predict orders of magnitude more dark matter subhalos within the virial radii of Milky Waymassed galaxies than are observed as luminous satellites of such systems (Moore et al. 1999; Klypin et al. 1999; Wadepuhl \& Springel 2011). Another aspect of the missing satellites problem is the discrepancy between the masses of the most massive predicted subhalos and

\footnotetext{
${ }^{1}$ Racah Institute of Physics, The Hebrew University, Jerusalem, Israel 91904; adizolotov@gmail.com

2 Department of Astronomy, University of WisconsinMadison, 475 N. Charter St., Madison, WI 53706 USA

${ }^{3}$ Department of Astronomy, Haverford College, 370 Lancaster Ave, Haverford, PA 19041 USA

4 Astronomy Department, University of Washington, Box 351580, Seattle, WA 98195 USA

${ }^{5}$ Oxford Astrophysics, University of Oxford, Denys Wilkinson Building, Keble Road, Oxford OX1 3RH

${ }^{6}$ Department of Astronomy/Steward Observatory, 933 North Cherry Ave, Tucson, AZ 85721 USA

${ }^{7}$ Department of Astronomy and Astrophysics, University of California, Santa Cruz, CA 95064, USA

${ }^{8}$ Department of Physics and Astronomy, McMaster University, Hamilton, Ontario L88 4M1, Canada
}

the most massive observed satellites (Moore et al. 1999; Klypin et al. 1999; Bovlan-Kolchin et al. 2011, 2012). Simulated $\sim 10^{12} M_{\odot}$ halos consistently have several subhalos that are too massive and too dense to host the most luminous dwarf spheroidal (dSph) satellites of the Milky Way (Bovlan-Kolchin et al. 2011, 2012; Wolf \& Bullock 2012; (Havashi \& Chiba| 2012). This appears to provide a new challenge for the CDM paradigm on small scales, because the most massive subhalos of an $\mathrm{L}^{\star}$ galaxy should not be devoid of stars.

The tension between the predicted and observed inner densities of the Milky Way's dSph satellites is reminiscent of the longstanding tension between the predicted and observed shapes of the central density profiles of galaxies (known as the "core/cusp problem"). The steep inner density profiles and concentrations predicted for dark matter halos and their satellites (Navarro et al. 1997; Lia et al. 2000; Dekel et al. 2003b. a; Reed et al. 2005; Springel et al. 2008; Madau et al. 2008; Navarro et al. 2010; Macciò et al. 2009) are inconsistent with those observed in isolated field galaxies (Persic et al. 1996; van den Bosch et al. 2000; de Blok et al. 2001; de Blok \& Bosma| 2002; Simon et al.|2003; Swaters et al. 2003; Weldrake et al. 2003; Kuzio de Narav et al. 2006; Salucci et al. 2007; Gentile et al. 2007; Spano et al. 2008; Trachternach et al. 2008; de Blok et al. 2008; Donato et al. 2009; Oh et al. 2011; Del Popolo 2012), and observed in satellites (Klevna et al. 2002, 2003; Mashchenko et al. 2005; Goerdt et al. 2006; Strigari et al. 2006; Gilmore et al. 2007; Walker et al. 
2009; Strigari et al. 2010; Walker \& Peñarrubia 2011; Jardel \& Gebhardt 2012; Wolf \& Bullock 2012; Havashi \& Chiba 2012; Salucci et al. 2012). This inconsistency is independent of whether the density slope in simulations follows $\log (\rho) \propto \gamma \log$ (radius), with $1.0<\gamma<1.5$ (i.e., a NFW profile, Navarro et al. 1997), or a power-law slope (i.e., an Einasto profile, Navarro et al. 2010).

Baryonic processes have often been proposed to address the apparent discrepancies between observations and the predictions of DM-only simulations. Within the CDM paradigm, the missing satellite problem is likely reconciled through reionization, which is expected to suppress star formation in subhalos with maximum circular velocity, $v_{c}$, at infall $<30 \mathrm{~km} / \mathrm{s}$ Quinn et al. 1996; Thoul \& Weinberg 1996; Navarro \& Steinmetz 1997; Gnedin 2000; Hoeft et al. 2006; Okamoto et al. 2008; Madau et al. 2008), and supernova feedback further suppressing star formation at the more massive subhalo end (Dekel \& Silk 1986; Benson et al. 2002; Dekel \& Woo 2003; Governato et al. 2007; Busha et al. 2010). When combined with observational incompleteness effects (Simon \& Geha 2007; Tollerud et al. 2008; Walsh et al. 2009; Koposov et al. 2009), it is likely that baryonic effects can bring the predicted number of subhalos in line with the observed number of satellites. Yet even if the correct number of satellites can be reproduced, CDM still predicts that the most massive satellites today are more massive and more dense than observed for Milky Way satellites (Bovlan-Kolchin et al. 2012; Wolf \& Bullock 2012; Hayashi \& Chiba 2012).

Baryons have also been invoked to reconcile the cusp/core problem in CDM. The predicted cuspy profiles of dark matter halos may not be physically realistic because the effect of energetic feedback from supernovae $(\mathrm{SNe})$ can reduce the baryonic (Dekel \& Silk 1986; Maller \& Dekel 2002; Dekel et al. 2003a; Brook et al. 2011; Guedes et al. 2011) and DM mass at the centers of galaxies (Navarro et al. 1996; Read \& Gilmore 2005; Mashchenko et al. 2006, 2008; Governato et al. 2010; Pasetto et al. 2010; de Souza et al. 2011; Cloet-Osselaer et al. 2012; Macciò et al. 2012; Pontzen \& Governato 2012; Governato et al. 2012; Teyssier et al. 2012; Ogiya \& Mori 2012). Importantly, there is not yet a unifying baryonic solution that solves the missing satellites, cusp/core, and massive subhalo problems simultaneously.

In fact, unlike the missing satellites problem and the core/cusp problem, solutions to reconcile the predicted massive subhalos with observed satellites have not yet addressed the effects of baryons. For example, studies have concluded that the excess of predicted massive subhalos around Milky Way-mass galaxies may disappear if satellites are modelled with Einasto (versus NFW) density profiles, or if the Milky Way's true virial mass is $\sim 8 \times 10^{11} \mathrm{M}_{\odot}$, rather than $\sim$ $10^{12} \mathrm{M}_{\odot}$ (Di Cintio et al. 2012; Vera-Ciro et al. 2012; Wang et al. 2012). It is not yet clear whether these solutions are themselves sufficient to fully reconcile observations with CDM-based models, and alternative cosmological models (e.g., warm or self-interacting dark matter) have also been proposed to explain observations (Alam et al. 2002; Strigari et al. 2007b; Lovell et al.
2012; Vogelsberger et al. 2012; van den Aarssen et al. 2012).

The inclusion of baryonic physics thus remains a glaring gap in predictions for the properties of dwarf satellites. Here we investigate whether the inclusion of energetic feedback from stars and supernovae, which has been shown to reduce the central dark matter densities of simulated field galaxies, can also reduce the predicted central densities of luminous satellites around Milky Way-mass galaxies. One reason that baryonic effects have not been studied in detail in satellite galaxies is because including baryons in cosmological simulations makes them much more computationally expensive. Previous work Governato et al. 2010; Guedes et al. 2011; Pontzen \& Governato 2012) demonstrated that simply achieving high resolution is not enough to reduce the central concentration of dark matter in galaxies. Instead, it is necessary to also adopt a physically motivated model in which star formation is limited to high density peaks, with densities comparable to the average densities in a giant molecular cloud. This model allows highly overpressurized regions to form when supernova energy is introduced to the high density surrounding gas, and the resulting hot bubble of gas flattens the central potential, leading to irreversible expansion of dark matter orbits as well as driving a wind (Pontzen \& Governato 2012).

The implications of such feedback may be all the more important when predicting properties of the satellites of larger galaxies (such as the Milky Way's own dwarf satellite population), because any effect imprinted on the central densities of the dwarf galaxies by baryons could be exacerbated during the dwarf galaxies' tidal evolution around their eventual host. The tidal effects of a disk are expected to be increasingly stronger as the central density profile of satellites becomes shallower (Taylor \& Babul 2001; Stoehr et al. 2002; Havashi et al. 2003; Read et al. 2006a; Peñarrubia et al. 2010). A proper prediction for both the total number and the internal structure of the Milky Way and M31's dwarf galaxies may thus require an accurate treatment of baryonic feedback. Only a few DM + baryon studies have focused on the satellite population of a Milky Way-massed galaxy, but at much lower resolutions than have been achieved in DM-only simulations, making comparisons with DM-only predictions for inner density profiles particularly difficult (see, however, Di Cintio et al. 2012; Parry et al. 2012).

In this paper, we compare DM-only and Smooth Particle Hydrodynamics (SPH) simulations of two Milky Way-massed galaxies run in a cosmological context to $z=0$. In fact, the simulations used in this work adopt a model that has already been shown to alleviate some of the small scale problems of CDM Governato et al. 2010; Oh et al. 2011; Brook et al. 2011; Macciò et al. 2012; Pontzen \& Governato 2012; Governato et al. 2012). This model is capable of forming bulgeless dwarf disk galaxies that have "cored" dark matter density profiles (i.e., shallower inner density slopes than predicted by CDM simulations without baryons, Governato et al. 2010, 2012; Oh et al. 2011). While the previous studies have focused on isolated field galaxies, this paper examines how this same model affects the satellite population of Milky Way-mass galaxies.

This paper is organized as follows. In Section 2 we de- 
scribe the simulations used in this work, as well as our method of selecting the satellite sample. In Section 3 we focus on the $z=0$ density and circular velocity profiles of SPH satellites, and compare to their DM-only counterparts. Section 4 explores the role of supernovae feedback and tidal stripping on the mass dependent evolution of satellites, from high redshift to $z=0$. In Section 5 we propose an update to the standard treatment of satellites in DM-only models. We summarize our results and conclude in Section 6 .

\section{SIMULATIONS}

The high-resolution N-body + SPH simulations used in this paper were run with GASOLINE (Wadsley et al. 2004). The two halos studied in this paper were initially selected from a uniform resolution, DM-only, 50 comoving Mpc box. The initial conditions for this box were generated assuming a WMAP Year 3 cosmology (Spergel et al. 2007): $\Omega_{m}=0.24, \Omega_{\Lambda}=0.76, H_{0}=73 \mathrm{~km}$ $\mathrm{s}^{-1}, \sigma_{8}=0.77$. The two halos $\left(\mathrm{h} 277, \mathrm{M}_{\text {vir }}=7 \times 10^{11} M_{\odot}\right.$, and $\left.\mathrm{h} 258, \mathrm{M}_{v i r}=8 \times 10^{11} M_{\odot}\right)$ were selected based on their $z=0$ mass and merging histories. We define virial mass relative to critical density, $\rho_{c}$, using $\rho / \rho_{c}=100$ at $z=0$ following Gross (1997). Galaxy h277 was selected to have a quiescent merger history, with its last major merger occurring at $z \sim 3$, while h258 has a binary merger at $z=1$. Each of h277 and h258 was resimulated at higher resolution (and with gas particles) using the volume renormalization technique (Katz \& White 1993). This approach simulates only the region within a few virial radii of the primary halo at the highest resolution, while still maintaining the large volume at low resolution in order to account for the large scale tidal field that builds angular momentum in tidal torque theory (Peebles 1969; Barnes \& Efstathiou 1987). The high resolution simulations were run from $z=150$ to $z=0$.

N-body $+\mathrm{SPH}$ volume renormalized simulations of both h277 and h258 have been studied in previously published work. For example, h258 was the focus of how a large disk can regrow by $z=0$ after a low $z$ major merger in Governato et al. (2009). The results in this paper are based on the same initial conditions as those previous studies, but simulated at a higher resolution that allows for the inclusion of new physics, discussed below. The spline force softening of the high resolution regions of both h277 and h258 is 174 pc. High resolution dark matter particles have masses of $1.3 \times 10^{5} M_{\odot}$, while gas particles start with $2.7 \times 10^{4} M_{\odot}$. Star particles are born with $30 \%$ of the mass of their parent gas particle (i.e., a maximum initial mass of $8100 M_{\odot}$ ), and lose mass through $\mathrm{SNe}$ and stellar winds. Each of these galaxies has roughly 5 million DM particles within the virial radius at $z=0$, and more than 14 million particles (dark matter + gas + stars) total.

The mass and force resolution of these runs is comparable to the "Eris" simulation (Guedes et al. 2011), one of the highest resolution $\mathrm{N}$-body $+\mathrm{SPH}$ cosmological simulations of a Milky Way-mass galaxy run to date. However, unlike Eris, we take advantage of the increased simulation fidelity that sufficiently resolves the high density regions where stars form $\left(\rho \sim 100 \mathrm{amu} / \mathrm{cm}^{3}\right)$ to alter the gas cooling and star formation prescription to include metal line cooling and $\mathrm{H}_{2}$. The inclusion of metal line cooling allows much more gas to cool to the central re- gions of the galaxy (Christensen et al., in prep.) and become fuel for star formation. However, the star formation in the new model is tied directly to the local $\mathrm{H}_{2}$ abundance, which is regulated by the gas metallicity and the ability of the gas to self-shield, in accordance with observational results (Lerov et al. 2008; Bigiel et al. 2008; Blanc et al. 2009; Bigiel et al. 2010; Schruba et al. 2011). The $\mathrm{H}_{2}$ prescription implemented here is described in detail in Christensen et al. (2012). Briefly, it includes both gas-phase and dust grain formation of $\mathrm{H}_{2}$, and destruction of $\mathrm{H}_{2}$ via photodissociation by Lyman-Werner radiation from nearby stellar populations. Before the implementation of the $\mathrm{H}_{2}$ model, gas particles were required to be above a set density threshold and below a set temperature before they could form stars. Tying star formation to molecular gas eliminates the need to set a density threshold above which stars can form, as it ensures that stars form at high densities. A gas particle must be colder than $1000 \mathrm{~K}$ before it can spawn a star particle.

Other aspects of the cooling physics and energy feedback from supernovae remain unchanged in these simulations. Shen et al. (2010) describes the primordial and metal-line cooling used, as well as the diffusion of metals that captures the effect of the turbulent interstellar medium on metal mixing. A uniform UV background turns on at $z=9$, mimicking cosmic reionization following a modified version of Haardt \& Madau (2001). Star particles are born with a Kroupa initial mass function (Kroupa et al. 1993). Each SN deposits $10^{51}$ ergs of thermal energy within a "blastwave" radius calculated following Ostriker \& McKee (1988), with cooling turned off in the affected region for a time that corresponds to the expansion phase of the SN remnant, and described in detail in Stinson et al. (2006). As described in Stinson et al. (2006), supernova type Ia and II yields are adopted from Thielemann et al. (1986) and Woosley \& Weaver (1995), respectively, and implemented following Raiteri et al. (1996). This prescription was shown to reproduce the observed stellar mass - metallicity relation for galaxies as a function of redshift (Brooks et al. 2007; Maiolino et al. 2008).

We demonstrate in this paper that including baryons in these simulations can dramatically alter the satellite evolution in comparison to the dark matter only case. Hence, we also have DM-only runs for the two halos described above. The force resolution and $z=0$ properties (i.e., number of DM particles and halo mass) of these DM-only halos are identical to the SPH runs, though the mass of the dark matter particles in the SPH runs is lower by a factor of $\left(1-f_{b}\right)$, where $f_{b}$ is the cosmic baryon ratio, $\Omega_{b} / \Omega_{m}$, and is 0.175 for the adopted cosmology.

\subsection{Satellite Selection \& Luminosity Function}

Because the main purpose of this work is to study the properties of the satellites of Milky Way (MW)-mass galaxies, we explain our method for selecting satellites in this section. Halos and their subhalos are identified at each output time step (every 320 million years) using AHF (Knollmann \& Knebe 2009; Gill et al. 2004). For field galaxies, AHF adopts overdensities with respect to the critical density as a function of redshift from Gross (1997). At $z=0$ the virial radius of h258 and h277 are $240 \mathrm{kpc}$ and $230 \mathrm{kpc}$, respectively. 
TABLE 1

Satellite Properties

\begin{tabular}{lcccc}
\hline \hline Satellite ID & $\begin{array}{c}M_{V} \\
z=0 \\
(1)\end{array}$ & $\begin{array}{c}M_{H I}\left[M_{\odot}\right] \\
(2)\end{array}$ & $\begin{array}{c}M_{H I}\left[M_{\odot}\right] \\
\left.z_{\text {infall }}\right]\end{array}$ & $\begin{array}{c}z_{\text {infall }} \\
(3)\end{array}$ \\
\hline h258, sat1 & -13.7 & $3.5 \mathrm{e} 5$ & $1.5 \mathrm{e} 7$ & 0.6 \\
h258, sat2 & -12.8 & $3.4 \mathrm{e} 5$ & $8.9 \mathrm{e} 6$ & 0.9 \\
h258, sat3 & -12.6 & $737.5^{\mathrm{a}}$ & $6.5 \mathrm{e} 6$ & 2.1 \\
h258, sat4 & -13.3 & 0. & $3.4 \mathrm{e} 6$ & 1.3 \\
h258, sat5 & -13.0 & 0. & $5.7 \mathrm{e} 7$ & 2.1 \\
h258, sat6 & -11.3 & $1.2 \mathrm{e} 5$ & $2.6 \mathrm{e} 6$ & 1.0 \\
h258, sat7 & -12.1 & 0. & $9.1 \mathrm{e} 5$ & 1.2 \\
h258, sat8 & -9.0 & 0. & 0 & 1.5 \\
h258, sat9 & -9.9 & 0. & $2.2 \mathrm{e} 5$ & 1.8 \\
h258, sat10 & -8.8 & 0. & 0 & 1.3 \\
h258, sat11 & -10.7 & 0. & $1.4 \mathrm{e} 6$ & 1.0 \\
h258, sat12 & -9.5 & 0. & $5.4 \mathrm{e} 4$ & 2.1 \\
h258, sat13 & -10.1 & 0. & $7.3 \mathrm{e} 3$ & 1.2 \\
\hline & & & & \\
h277, sat1 & -14.7 & $3.2 \mathrm{e} 7$ & $2.2 \mathrm{e} 7$ & 0.02 \\
h277, sat2 & -13.9 & $3.8 \mathrm{e} 7$ & $3.7 \mathrm{e} 7$ & 0.2 \\
h277, sat3 & -14.3 & $5.1 \mathrm{e} 6$ & $3.8 \mathrm{e} 7$ & 1.5 \\
h277, sat4 & -13.4 & $1.3 \mathrm{e} 6$ & $3.0 \mathrm{e} 6$ & 0.9 \\
h277, sat5 & -10.4 & 0. & 0 & 1.4 \\
h277, sat6 & -10.6 & 0. & $9.0 \mathrm{e} 6$ & 1.6 \\
h277, sat7 & -9.9 & 0. & $1.4 \mathrm{e} 5$ & 1.6
\end{tabular}

Note. - Column (1) V-band magnitude, calculated based on the age and metallicity of the star particles and adopting the Starburst99 stellar population synthesis models of Leitherer et al. (1999) and Vázquez \& Leitherer (2005) for a Kroupa IMF (Kroupa 2001). Column (2) is the mass in HI gas in the satellite at $z=0$. Column (3) is the mass in HI gas in the satellite at infall. The redshift of infall is listed in column (4).

${ }^{a}$ Given the radial distance of this object from the center of the main halo that it orbits, this mass of $\mathrm{HI}$ would remain undetected by current $\mathrm{HI}$ observations. We therefore consider it a dSph analog.

We first find all of the subhalos that are within the $z=0$ virial radii of the two primary halos described above. We then reduce that list to only those subhalos that are luminous, with a minimum of 10 star particles. This list of subhalos is likely to include some subhalos that have undergone a large amount of dark matter stripping, but Brooks et al. (2007) showed that the star formation histories (SFHs), and hence stellar masses, of these simulations converges only when the halo has more than $\sim 3500$ DM particles. Hence, we then trace back the primary progenitor halo for each of the $z=0$ subhalos. To do this, we successively identify the halo at each higher $z$ step that contains the most DM mass of the lower $z$ halo. We verified that all of our $z=0$ luminous satellites have had more than 7000 DM particles within their virial radius at some point in the halo's history, ensuring that their stellar masses are robust to resolution effects. We exclude bright, Magellanic-like satellites with $M_{V}<-15$ from this analysis, which removes from each simulation one bright satellite. However, we make no cut on satellite morphology at $z=0$, so that the final list of subhalos includes both gas-free and gas-rich satellites.

Table 1 lists some of the properties of the satellites studied in this paper. We note that h258 has 13 luminous satellites and h277 has 7 satellites, despite their similar halo masses. This demonstrates that the number of satellites (and particularly the number of highest mass satellites) is very stochastic at a fixed parent halo mass (Vera-Ciro et al. 2012; Sawala et al. 2012).

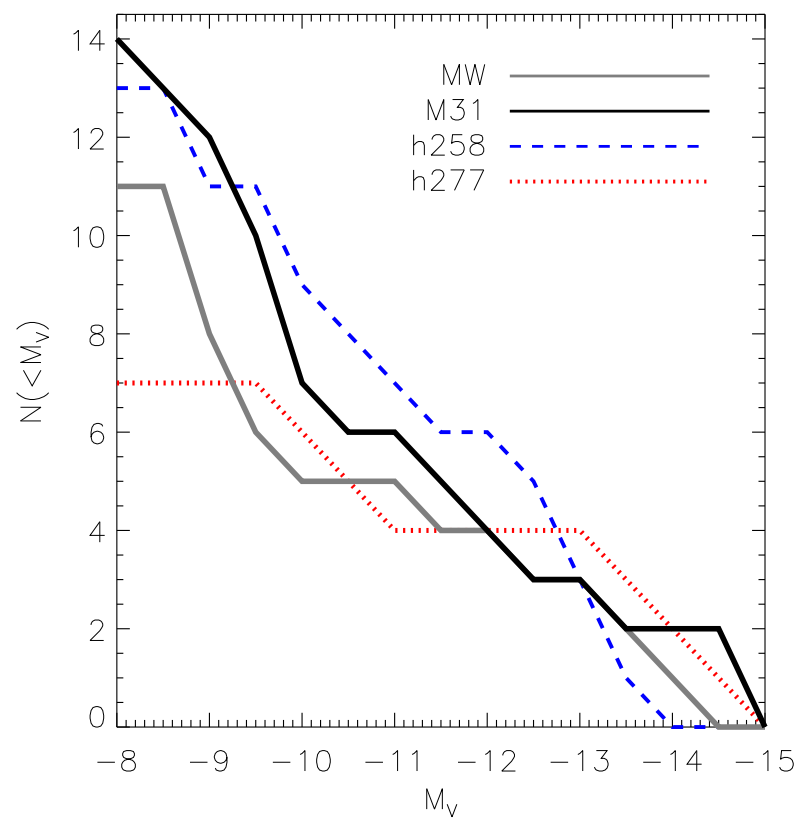

FIG. 1. - The satellite luminosity function for our two simulated galaxies compared to the Milky Way and M31 satellite luminosity functions. The simulated luminosity functions contain all satellites listed in Table 1.

The orbital evolution of every satellite was traced with respect to the parent halo. Although some satellites may enter the virial radius of the parent halo and exit again, we identify the infall redshift as the time at which the satellite first enters the parent halo's virial radius. The infall times are listed in Table 1 , and in all cases the infall redshift is at $z<3$ (see also Zentner \& Bullock 2003; Geen et al. 2012).

Figure 1 shows the satellite luminosity functions for our two simulated galaxies compared to that of the Milky Way and M31. The simulation luminosity functions include both gas-free and gas-rich satellites. The MW and M31 luminosity functions include all satellites fainter than $M_{V}=-15$, including Sagittarius and Canis Major in the case of the MW, as compiled in McConnachie (2012). Our simulated gas-free satellite sample spans the luminosity range of the Milky Way's classical dwarf spheroidals, $-13.3<M_{V}<-8.8$, from Fornax to Canes Venatici I $\left(M_{V}=-13.4\right.$ and -8.6 , respectively, McConnachie 2012).

We stress that no attempt was made to explicitly match the classical dSph luminosity range, although our final sample does. Instead, the deposition of SN energy combined with $\mathrm{H}_{2}$-based star formation was implemented to reproduce the stellar mass of the parent halo at the given halo mass. While past simulations have overproduced stellar mass at a given halo mass (Zolotov et al.|2009; Guo et al.|2010; Sawala et al. 2011; Brooks et al. 2011; Leitner 2012), it has been suggested that restricting star formation to depend on the mass in molecular gas (rather than total gas mass) will alleviate this problem, particularly at high $z$ where metallicities are low and formation of $\mathrm{H}_{2}$ on dust grains is reduced (Robertson \& Kravtsov 2008; Gnedin et al. 2009; Gnedin \& Kravtsov 2010; Krumholz \& Dekel 2011; 
Feldmann et al. 2011; Kuhlen et al. 2012). Munshi et al. (2012) demonstrate that the parent halos in this paper match the observed $z=0$ stellar mass to halo mass relation (Guo et al. 2010; Behroozi et al. 2010; Moster et al. 2010; Conrov \& Wechsler 2009; Yang et al. 2011; Neistein et al. 2011; Leauthaud et al. 2012). Also, since star formation becomes metallicity dependent in this $\mathrm{H}_{2}$-based scheme, it lowers the star formation efficiency in the low mass, low metallicity satellites studied in this paper, preventing them from overproducing stars. Governato et al. (2012) previously demonstrated that the stellar masses and total masses at $<1 \mathrm{kpc}$ for simulated field galaxies in the same stellar mass range as the satellite galaxies in this paper are in excellent agreement with the observational data.

It is possible that some of the gas-rich satellites included in this work may be unphysically gas-rich, because SPH does not accurately model small-scale hydrodynamical instabilities that can lead to enhanced gas stripping from satellites (Agertz et al. 2007; Mitchell et al. 2009). However, Weisz et al. (2011) have recently shown that dSphs with $M_{B}<-10$ have extended SFHs, suggesting that the majority of observed gas-free satellites at $z=0$ had gas until recently. Thus, despite the fact that some of our satellites in the luminosity range of the MW's dSphs have gas at $z=0$, their SFHs are consistent with those observed.

\subsection{Matching SPH \& DM-only Satellites}

We have identified the subhalos in the DM-only runs that correspond to each of the luminous satellites in the SPH runs. This matching is performed by requiring that SPH and DM-only satellites have the same properties before infall, as well as similar orbits after infall, in order to be considered a pair. We do this by first compiling a list of candidate matches by identifying the DM-only halos with the best virial mass and position matches at $\mathrm{z}=3$ (prior to infall and any stripping) to the SPH halos. We then identify the infall time and trace the full orbital histories of the candidate halos around the primary galaxy. In order to be considered a match a DM-only halo must have a similar orbital history around the primary galaxy as the SPH satellite. For three of our satellites, an exact match was not found, and therefore these three satellites are excluded from any direct comparison of matched subhalos in the remainder of the paper.

\section{REDSHIFT 0 RESULTS}

In this Section, we present the DM density profiles of satellites of MW-massed disk galaxies at present day. In order to study the effect that star formation has had on the internal structure of the satellites, we have divided the satellite sample by stellar mass at $z=0$. "Luminous" satellites have stellar mass greater than $10^{7} M_{\odot}$, while "low-luminosity" satellites are those with stellar masses less than $10^{7} M_{\odot}$. We further subdivide the luminous satellites into categories of gas-rich and gas-free. We do this in order to distinguish between simulated satellites that would match the MW's dSph population, which are all gas-free dwarfs, and simulated satellites that more closely resemble the Magellanic clouds, which are gasrich. All of the satellites categorized as "low luminosity" are gas-free.
In Figure 2 we show the dark matter density profiles, $\rho(r)$, for a subsample of the SPH satellites (black solid lines) and their DM-only counterparts (blue dashed lines). Each panel in this figure shows satellites from one of the three categories we have defined - the three most luminous gas-rich satellites are shown in the left panel, the three most luminous and gas-free galaxies in the middle panel, and the three least luminous satellites in the right panel. Figure 2 shows that the most luminous and gas rich satellites in the SPH runs are less dense and have flatter inner density profiles than their DM-only counterparts. While the luminous gas-free satellites (middle panel) do not appear strongly cored, a direct comparison between the SPH and DM-only matches shows that the $\mathrm{SPH}$ runs do have a more shallow inner profile. Importantly, these gas-free, luminous satellites have dramatically lower densities overall than their DM-only counterparts. At the lowest luminosity end, however, the satellites in the SPH and DM-only runs tend to have comparable density profiles (slope and normalization). Hence, the process that lowers the density in our SPH satellites is more effective at the high stellar mass end than at the low stellar mass end. We demonstrate that this process is related to the $\mathrm{SFH}$ of each satellite in the next section. We note that because the DM particle masses in the SPH run are lower than in the DM-only run by the cosmic baryon factor, $f_{\text {bar }}$ (i.e., these particles have been split into DM and gas in the initial conditions), we have reduced the DM-only densities by $f_{\text {bar }}$ in order to make a direct comparison in this figure.

Figure 2 demonstrates that, even at moderate dSph galaxy luminosities $\left(M_{V} \lesssim-12\right.$, similar to Leo I or And II), baryonic processes result in an expanded and shallower central dark matter distribution than predicted by DM-only simulations. Even when the cored 9 density profiles of our simulated dwarfs do not have a flat slope $(\gamma=0)$, the absolute values of density in the central regions are still dramatically reduced compared to expectations from a DM-only simulation. We conclude that DM-only simulations, or models based on an assumption of adiabatic contraction, make physically incorrect predictions for the central $(<1 \mathrm{kpc})$ masses of dwarf galaxies more luminous than Leo I $\left(M_{V}=-11.9\right)$. Such models should therefore be used with caution when used to interpret the observed dynamics of dwarf satellites in a cosmological context.

The general conclusions discussed in this paper are independent of resolution effects, as is discussed in detail in the Appendix. In the remainder of the paper we will often compare the circular velocity, $v_{c}$, values in the $\mathrm{SPH}$ and DM runs at $1 \mathrm{kpc}$. As will be seen in the next section, the $v_{c}$ values at $1 \mathrm{kpc}$ show the dramatic effect that baryonic physics has on the SPH runs, but avoids biasing this value due to convergence issues at smaller radii. More importantly, the $v_{c}$ values at $1 \mathrm{kpc}$ between the SPH and DM-only runs are in excellent agreement in our low-mass subhalos for which baryons do not dramatically alter the evolution. The convergence of SPH and DM-only results in these lower mass halos (which, by definition, contain fewer particles and are less resolved than their high mass counterparts) demonstrates that there

${ }^{9}$ In the rest of this paper, we will use the term "cored" to refer to all slopes shallower than predicted by DM-only simulations alone. 

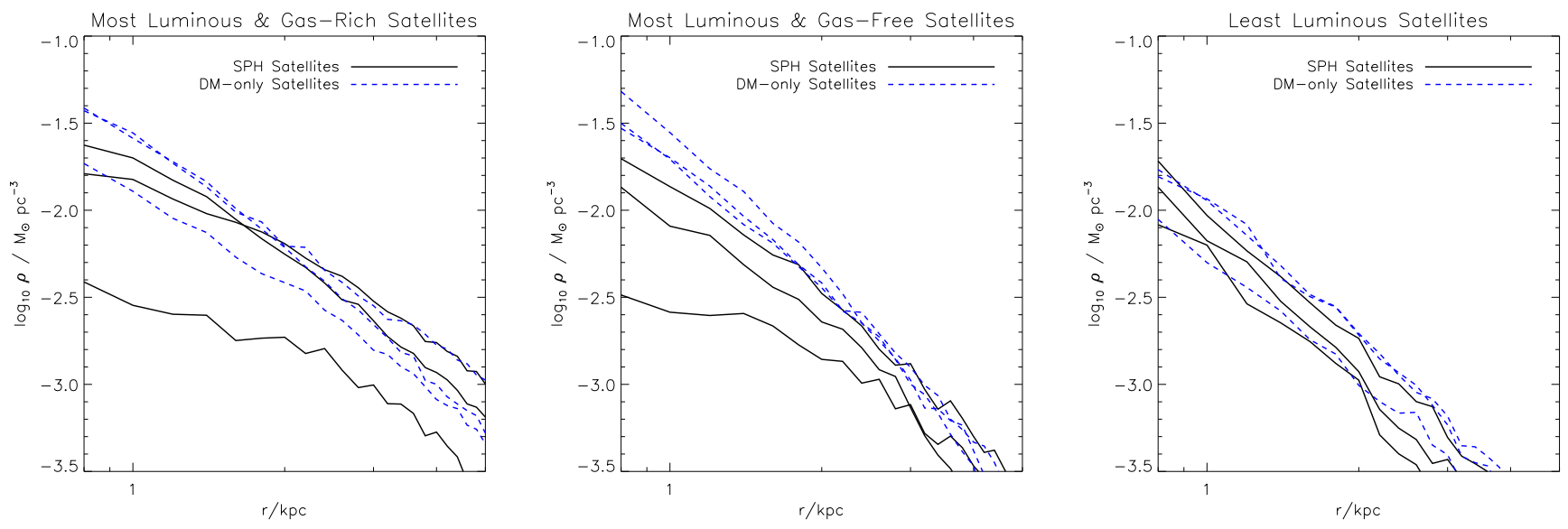

FIG. 2. - The DM density profiles of SPH satellites (solid black lines) and their DM-only counterparts (blue dashed lines) at $z=0$. The left panel shows the three most luminous SPH satellites, which are also still gas-rich at $z=0$. The middle panel shows the three most luminous, gas-free satellites, and the right panel shows the three least luminous SPH satellites. Luminous SPH satellites have significantly shallower central density profiles than DM-only satellites, while low-luminosity SPH satellites retain central density cusps similar to their DM-only counterparts.

are no spurious numerical effects introduced by the lower mass baryonic particles in the SPH runs.

\section{MASS DEPENDENT EVOLUTION OF SATELLITES WITH BARYONS}

In this Section, we study the evolution of the satellites to understand the processes that lead to the lower concentration of mass at $z=0$ in the SPH satellites. We first focus on the evolution at high redshift, and demonstrate that DM core creation occurs in the most luminous satellites prior to their infall. After infall, we show that tidal stripping effects exacerbate the mass discrepancy between SPH satellites and their DM-only counterparts.

\subsection{The Impact of Baryons Before Infall}

We now examine the evolution of $v_{c}$ from high $z$ to infall for all of the satellites in our sample. The top panel of Figure 3 shows the change in the DM contribution to $v_{c}$ at $1 \mathrm{kpc}$ between $\mathrm{SPH}$ satellites and their DM-only counterparts at infall, as a function of the stellar mass of the SPH satellites at infall. It can be seen in this panel that satellites that have formed more stars prior to infall (those with $\mathrm{M}_{\star}>10^{7} M_{\odot}$ at infall) undergo a significant decrease in DM mass interior to $1 \mathrm{kpc}$, in comparison to their matched counterparts in the DM-only runs. The DM rotation curves of these luminous satellites are therefore $2-16 \mathrm{~km} / \mathrm{s}$ lower than those of DM-only satellites. (Masses in the DM-only run have been reduced by $f_{\text {bar }}$ for a direct comparison.) We conclude that baryonic effects lower the central DM densities, and hence lower the central DM circular velocity, of massive satellites prior to infall.

The bottom panel of Figure 3 shows the total change in $v_{c}$ at $1 \mathrm{kpc}$ between SPH satellites and their DMonly counterparts at infall. This panel shows that the overall reduction in total $v_{c}$ is is not as strong as the reduction in DM $v_{c}$. Although the DM mass has been reduced for satellites with $\mathrm{M}_{\star}>10^{7} M_{\odot}$, the presence of baryons contributes to the central masses in such a way that the overall mass is not necessarily reduced compared to the DM-only case prior to infall. Note that this is not adiabatic contraction, in which the central DM densities are increased due to the cooling of baryons, as we have

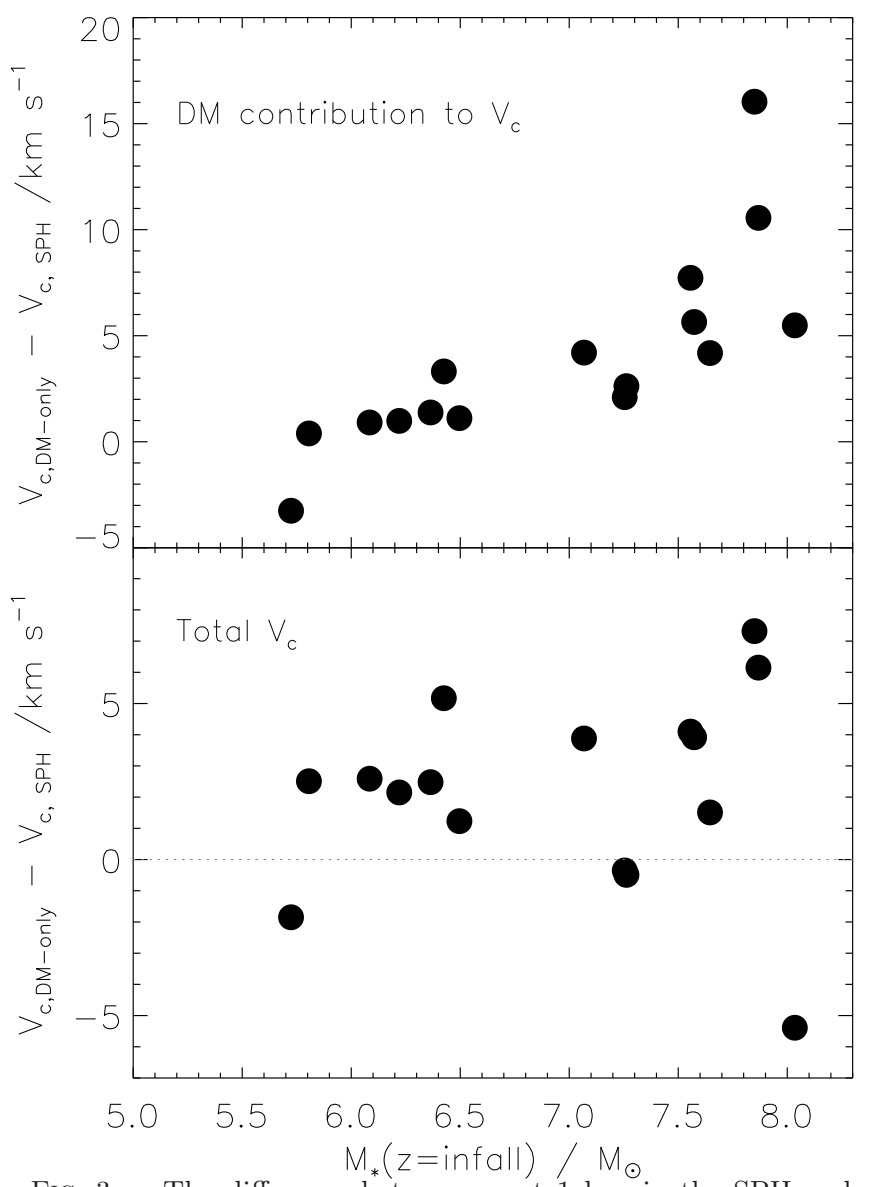

FIG. 3.- The difference between $v_{c}$ at $1 \mathrm{kpc}$ in the SPH and DM-only runs at infall, as a function of the stellar mass in the SPH satellite at infall. Top panel: The difference in the DM contribution to $v_{c}$ at $1 \mathrm{kpc}$ for matched SPH and DM-only subhalos. Bottom panel: The difference in total $v_{c}$ at $1 \mathrm{kpc}$

just demonstrated that the baryonic runs have comparable or lower DM densities to the DM-only runs. We demonstrate below that once gas is stripped from the SPH satellites after infall, the reduction seen in the DM masses and the reduction in total mass are in agreement. The global trend of $\sim 3 \mathrm{~km} / \mathrm{s}$ reduction in total $v_{c}$ in the 

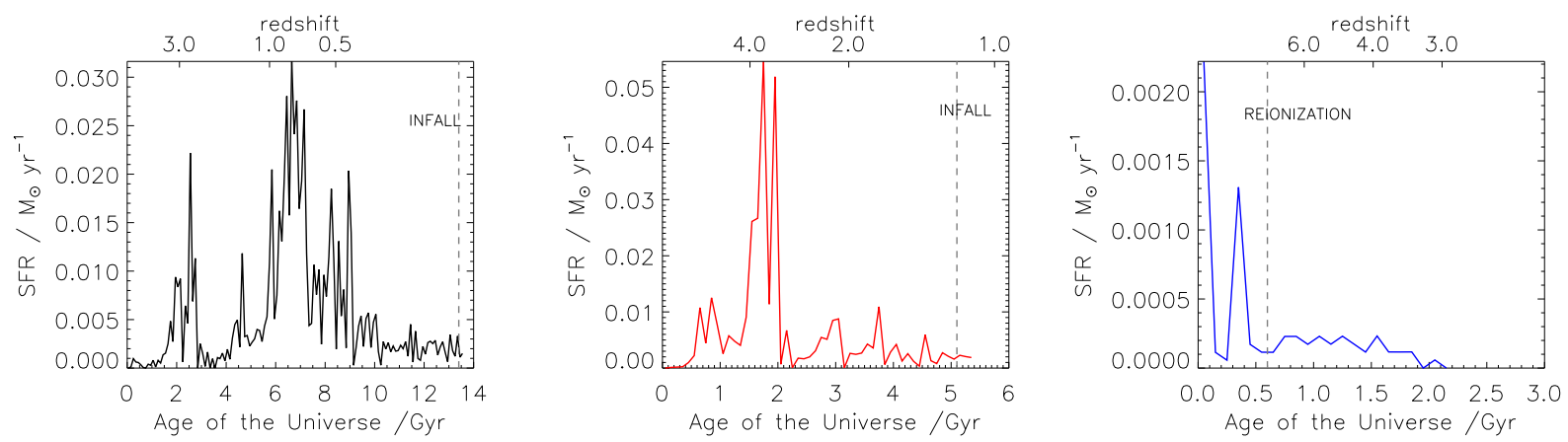

FIG. 4. - The star formation histories of three SPH satellites. Note the different range for the axes. At infall, the satellites in black (left panel), red (middle panel), and blue (right panel) have virial masses of $7.9 \times 10^{9}, 2.7 \times 10^{9}, 0.6 \times 10^{9} M_{\odot}$, respectively, and infall times of $z=0.02,1.3,1.5$. In the first two panels, the vertical dashed line marks $z$ =infall onto the parent halo. In the final panel, $z=$ infall lies beyond the range of the x-axis, which is truncated before infall for clarity. However, the vertical dashed line in the right panel indicates the onset of reionization (i.e., when the uniform UV background turns on).

$\mathrm{SPH}$ runs is due to gas lost in either SNe-driven outflows or reionization.

Governato et al. (2012) also found that DM core creation varied as a function of mass, for isolated field galaxies. These authors have shown that the creation of DM density cores due to $\mathrm{SNe}$ driven outflows is common in galaxies with $M_{\star}>10^{7}$ at $z=0$. For lower luminosity galaxies, their work finds that SF and its associated feedback are not efficient enough to have flattened a galaxy's steep DM density profile. We find that this mass threshold above which feedback becomes effective is the same in satellite galaxies as well, despite the fact that core formation does not continue after infall.

Several theoretical models have shown how outflows and galactic fountains can lead to the flattening of DM density cores in dwarf galaxies (Navarro et al. 1996; Read \& Gilmore 2005; Pontzen \& Governato 2012; Governato et al. 2012). Rapid and frequent star formation episodes break the adiabatic approximation in the central kiloparsec of galaxies, transferring energy to the collisionless particles, and resulting in shallow DM density cores (Pontzen \& Governato 2012; Ogiva \& Mori 2012). An essential component to such DM-flattening scenarios is the ability to resolve the high density clumps where star formation takes place, i.e., overdensities comparable to giant molecular clouds (Robertson \& Kravtsov 2008; Saitoh et al. 2008; Tasker \& Brvan 2008; Ceverino \& Klypin 2009; Christensen et al. 2010; Colín et al. 2010). When star formation is limited to these high density peaks, energy deposited from $\mathrm{SNe}$ creates overpressurized regions of hot gas, driving outflows of SNe heated gas from the simulated galaxies. The $\mathrm{H}_{2}$ model adopted in these simulations includes self-shielding of cold gas, preventing heating from photoionization in dense regions with no young stars nearby. Self-shielding, combined with a low temperature cooling model, allows the already cool gas in the galaxy to cool even further, making the star formation even clumpier and the feedback therefore even more efficient (Christensen et al. 2012; Susa 2008, Christensen et al., in prep.).

When star formation is limited to high density peaks, as in this paper, the SNe feedback after a star forming event creates regions of overpressurized, hot gas that will shut down additional star formation for a period of time until the gas can cool and continue to form more stars.
This process leads to a bursty SFH. Examples of the resulting star formation histories for galaxies in this paper are shown in Figure 4. Dwarf galaxies in our highest luminosity range are massive enough to retain gas for an extended period of time, allowing them to undergo multiple star forming and gas loss events. Each event pushes successively more DM to larger orbits, gradually transforming a cuspy DM density profile into a flatter cored profile. Hence, extended SFHs that allow for multiple bursts of SF lead to effective DM core creation. At the lower end of the luminosity range of our satellites, the halos are less massive, with shallower potential wells. A few are low enough in mass that they lose their gas relatively early, though most maintain some gas at least until infall. The overall gas mass in these low mass subhalos is substantially reduced at early times, due to a combination of reionization and initial SF that ejects gas (see also Sawala et al. 2010). The remaining gas in the shallow potential well then has a difficult time reaching the densities required for star formation. The inability to continue forming stars in the lower mass, less luminous satellites prevents them from having bursts of star formation over extended periods, and they thus retain a steeper DM density profile.

If the mass trends described above are accurate, we should see these trends reflected concurrently in the SFHs of the galaxies and their reduction in central dark matter mass. To examine the evolution of the satellites as a function of mass, we focus on the progenitors of three satellites as examples, drawn from the three different categories represented in Figure 2 (gas-rich, gas-free and most luminous, gas-free and least luminous). Figure 4 shows the SFHs of these three representative satellites. The SFHs, particularly in the luminous satellites, are episodic with bursts, rather than smooth or constant. In Figure 5 we show the evolution of the DM contribution to $v_{c}$ at $1 \mathrm{kpc}$ in these same three galaxies. Each $v_{c}$ evolution curve is normalized to the $v_{c}$ value of the DM at infall, where the infall times are marked by vertical lines, color coded to each galaxy.

For the luminous satellites (shown in black and red in Figures 4 and 5), reductions in the DM contribution to $v_{c}$ at $1 \mathrm{kpc}$ can be seen following strong bursts of $\mathrm{SF}$ in these galaxies. For example, a DM core begins to form in the progenitor of the most luminous satellite (shown in black) near $z=1$, consistent with a large 


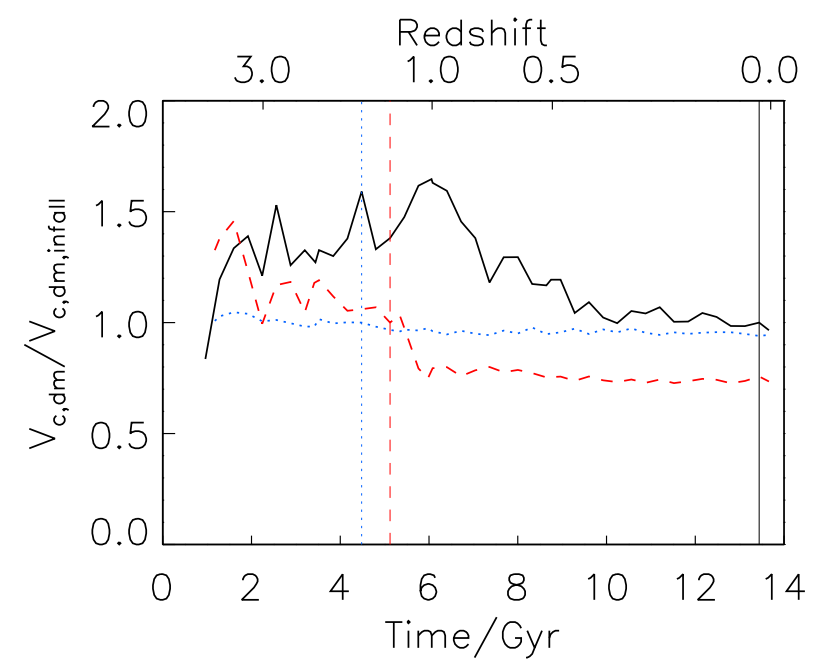

Fig. 5.- The DM contribution to $v_{c}$ at $1 \mathrm{kpc}$ in the three SPH satellites shown in Figure 4 as a function of time. The $v_{c}$ value at all times is normalized to the value at infall. A luminous gaseous (at $z=0$ ) satellite is in black (solid line), a luminous gas-free (at $\mathrm{z}=0$ ) satellite is in red (dashed line), and a low luminosity gas-free (at $\mathrm{z}=0$ ) satellite in blue (dotted line). Their respective infall times are shown by the vertical lines, with colors and linestyles according to galaxy as just described. In the two most luminous satellites, decreases in $v_{c}$ correspond to bursts of SF seen in Figure 4

number of repeated bursts of SF that begin at this time. Figure 5 indeed shows a significant decrease in the central DM mass associated with core formation beginning at $z \sim 1$. During periods of low SF, baryons have little to no impact on the DM structure of these satellites. For example, the satellite shown in red does not undergo any changes in the contribution of DM to its central $v_{c}$ between $z=3$ and infall, which is tied to the weak SF of this galaxy during this period.

The low luminosity, gas-free satellite (shown in blue in Figures 4 and 5) forms most of its stars prior to reionization. The gas supply of this satellite is strongly affected by reionization, with SF declining in the $\sim 3$ Gyr afterward, until gas is completely unbound from this halo at $z \sim 2$. The single, small burst of SF in this galaxy prior to reionization was not enough to alter the DM density profile, nor was the steady decline in SF between reionization and $z=3$. This is evidenced in Figure 5, which shows that the DM contribution to $v_{c}$ in the central $1 \mathrm{kpc}$ stays constant through the entire history of this galaxy, both prior to and after infall.

Finally, we verified that the SFHs of satellites are either completely truncated at infall, or SF continues at a low rate with no strong bursts of SF that are associated with core formation. Thus, we conclude that the dark matter core formation occurs prior to infall. The SFHs of the galaxies before infall are intimately tied to their DM density profiles, with multiple SF bursts required to create a DM core in the progenitor of a satellite (Read \& Gilmore 2005; Pontzen \& Governato 2012).

\subsection{Evolution After Infall}

In this Section, we focus on the evolution of the central mass of satellites after infall in order to explore whether the presence of baryons in galaxies leads to
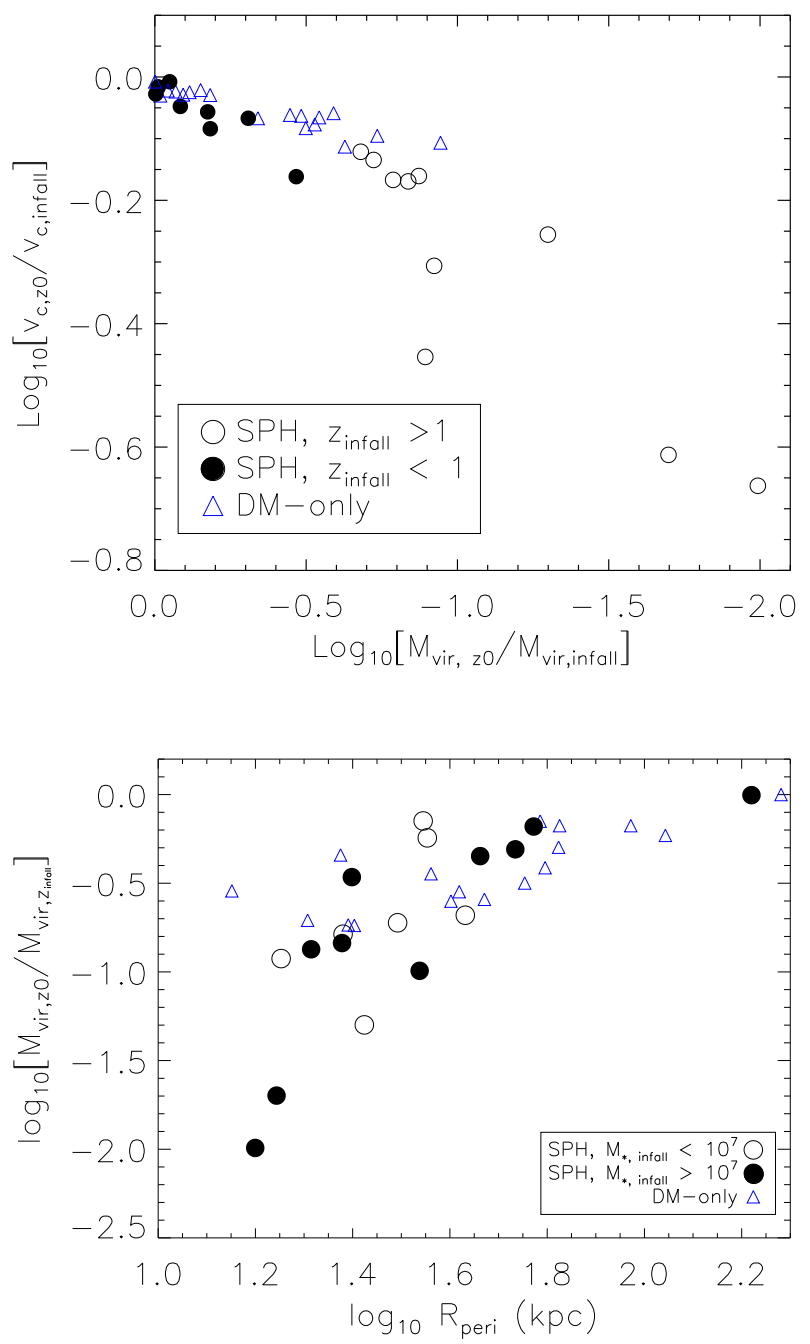

FIG. 6.- Top Panel: The change in $v_{c}$ at $1 \mathrm{kpc}$ between $z=$ infall and $z=0$ for the simulated satellites, as a function of the fraction of mass retained since infall. The SPH satellites (in circles) are divided by their infall time, and the matched DM-only counterparts are shown as blue diamonds. Bottom Panel: The fraction of mass retained after infall by satellites, as a function of the minimum pericenter radius in their orbital history. The SPH satellites (in circles) are divided by their stellar mass at infall time in this panel.

diverging tidal evolution between the SPH and DMonly cases. In tracing the orbits of our satellite sample, we found that some satellites undergo significant mass loss after accretion, with most of the mass loss occurring at pericenter passage where tidal effects are strongest. This is true in both the SPH and DM-only runs, a result that has been seen in many other studies (e.g., Maver et al.|2001; Dekel et al.|2003a; Havashi et al.|2003; Kazantzidis et al.|2004; Maver et al. 2006; Read et al. 2006b; Bovlan-Kolchin \& Ma 2007; Macciò et al.|2009; Choi et al. 2009; Klimentowski et al. 2010; Kazantzidis et al. 2011). However, most work to date on mass loss in satellites has excluded the effects of baryons. We demonstrate below that when a satellite's orbit is such that it is likely to undergo significant tidal stripping, SPH satellites will lose a substantially larger fraction of their central mass after infall compared to their DM-only counterparts. 
In the top panel of Figure 6 we show the change in circular velocity, between infall and $z=0$, at $1 \mathrm{kpc}$ for all simulated satellites, as a function of the fraction of mass retained since infall. Two important trends emerge in this figure. First, SPH satellites tend to undergo a larger decrease in their $v_{c}(1 \mathrm{kpc})$ than their DM-only counterparts after infall. We find that the relative reduction of $v_{c}$ between SPH and DM-only satellites is highly dependent on the orbital parameters of the satellites. For example, satellites accreted more than 6 Gyr ago $\left(z_{\text {infall }}>1\right)$ experience a reduction in their central circular velocities that is $11-62 \%$ more than the reduction in $v_{c}(1 \mathrm{kpc})$ experienced by their DM-only counterparts. Likewise, satellites on more radial orbits $\left(r_{p e r i}: r_{a p o}>8\right)$ undergo a reduction in $v_{c}$ that is $35 \%$ greater than their DM-only matches. On the other hand, SPH satellites accreted more recently $\left(z_{\text {infall }}<1\right)$ or on circular orbits $\left(r_{\text {peri }}: r_{\text {apo }}<2\right)$ have their $v_{c}$ reduced by only $0-13 \%$ more than their DM-only counterparts. A larger decrease in $v_{c}(1 \mathrm{kpc})$ will occur in some of the SPH satellites simply from gas loss (e.g., in ram pressure stripping). A comparison of the HI masses at infall to the $z=0 \mathrm{HI}$ masses in Table 1 demonstrates that significant amounts of gas are lost. In the vast majority of the satellites, however, the decrease in $v_{c}$ in the SPH satellite is significantly higher than can be accounted for by just loss of gas. The second trend seen in the top panel of Figure 6 is that only $\mathrm{SPH}$ satellites lose more than than $90 \%$ of their total mass 10 Overall, we find that SPH satellites lost $3-34 \%$ more of their virial mass than their DM-only counterparts after infall.

Peñarrubia et al. (2010, hereafter P10) used Nbody simulations to examine the role of different DM density profiles, as well as the role of a baryonic disk in the host, on the tidal evolution of satellites (see also Taylor \& Babul 2001; Stoehr et al. 2002; Havashi et al. 2003; Kazantzidis et al. 2004; Read et al. 2006a; Choi et al. 2009; D'Onghia et al. 2010; Romano-Díaz et al. 2010; Wetzel \& White 2010; Nickerson et al. 2011). Their models show that the presence of a baryonic disk in the primary galaxy results in a higher mass-loss rate for satellites at each pericentric passage, in comparison to a host with no disk. The influence of a baryonic disk is especially strong for satellites with shallow DM density profiles, with slopes of $0.0 \leq \gamma \leq 0.5$, resulting in a higher fraction of mass loss at each pericenter passage for the cored satellites. The combined effects of the host disk and a satellite's shallow DM density profiles in P10 becomes increasingly significant with increasing time after infall and for satellites on more eccentric orbits.

The greater reduction in central circular velocities and virial masses we find in our SPH satellites is due in part to the presence of a disk in the SPH host galaxies (the DM-only runs do not have a disk), which results in more efficient mass loss for the SPH satellites. Indeed, the three SPH satellites that have lost the most mass $(>90 \%)$, are all on orbits whose pericenters bring them

\footnotetext{
10 Subhalos must have lost $\sim 90 \%$ of their mass before stars, which are more tightly bound, begin to be stripped (Peñarrubia et al.2008). Only three satellites in our sample change their stellar mass by more than $20 \%$ between infall and $z=0$, and they are the same three that have lost more than $90 \%$ of their total mass.
}

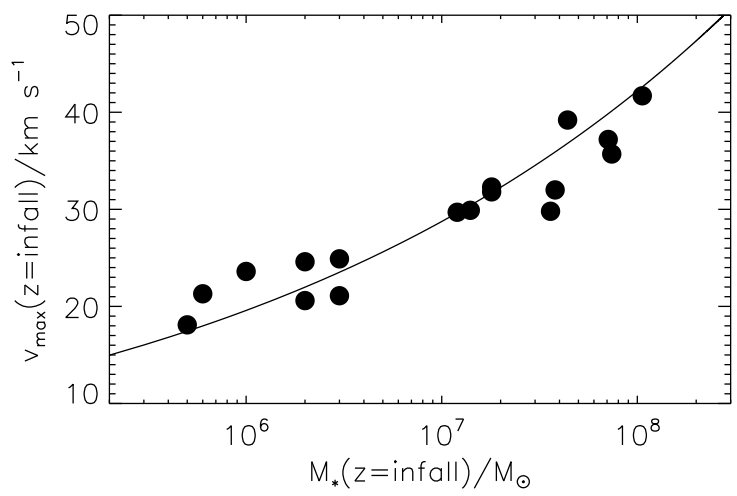

FIG. 7.- The $v_{\max }$ values of the SPH subhalos at infall as a function of their stellar mass at infall. The solid line shows the relation $\mathrm{M}_{*} \propto v_{\max }^{6}$, normalized at $M_{*}=10^{7} \mathrm{M}_{\odot}$, indicating how stellar mass increases with $v_{\max }$ in these simulations.

within the inner $30 \mathrm{kpc}$ of the galaxy, where the effects of the disk on mass loss are strongest. This can be seen in the bottom panel of Figure 6. Furthermore, the two satellites that lost the most amount of mass both had shallow DM density profiles at infall (with $M_{*}>10^{7} M_{\odot}$ at infall). As noted in P10, the effect of the disk should be more pronounced in the tidal evolution of cored satellites, since the disk can dominate the tidal field in the center of cored satellites, but not in the tightly bound cuspy halos. Indeed, our samples contains two SPH satellites one with a cored DM density profile and one with a cuspy DM density profile - that have similar infall times, orbital histories, and both have $R_{\text {peri }}<30 \mathrm{kpc}$. The $v_{c}$ at $1 \mathrm{kpc}$ of the cored SPH satellite was reduced by $78 \%$ after infall, while the cuspy SPH satellite underwent only a $44 \%$ reduction in its central $v_{c}$.

We conclude that if a satellite is likely to have undergone significant tidal stripping due to its orbital history, the existence of a baryonic disk in the host and a shallow inner DM density profile in the satellite will exacerbate the amount of mass lost.

\section{AN UPDATE TO THE THEORETICAL MODEL}

When using DM-only theoretical results to interpret the MW's dwarf satellite population, it is common to associate the DM-only subhalos that were the most massive at the time of their formation or accretion with the more luminous dSphs (Bullock et al. 2000; Kravtsov et al. 2004; Gnedin \& Kravtsov 2006; Strigari et al. 2007a; Koposov et al. 2009; Bovill \& Ricotti 2011; Simha et al. 2012), i.e., an abundance matching technique. This assignment is reasonable if we assume that the most massive halos will have the highest star formation rates, and therefore be the most luminous at $z=0$. Figure 7 shows that our simulated halos follow a trend of increasing stellar mass with halo mass (represented by $v_{\max }$ ) prior to infall, so that the most luminous galaxies at $z=0$ indeed correspond to the most massive galaxies at infall. We verified that the matched DM-only counterparts can be assigned to the most luminous satellites at infall in the SPH run. In these simulations, $\mathrm{M}_{*} \propto \mathrm{M}_{v i r}^{2}$ (see Governato et al. 2012). Because $v_{\max }$ roughly scales as $\mathrm{M}_{\text {vir }}^{1 / 3}$ (e.g., Klypin et al. 2011), $\mathrm{M}_{*} \propto v_{\max }^{6}$. This relation is shown as the solid line in Figure 7 normalized 
at $\mathrm{M}_{*}=10^{7} \mathrm{M}_{\odot}$. We note, however, that tidal stripping can substantially reduce the mass of some halos by $z=0$, introducing scatter into the tight relation seen in Figure 7 at infall.

While the assumption that stellar mass increases with halo mass is valid for these simulations, it cannot be assumed that the central dark matter mass distribution of the DM-only subhalos is the same as that of luminous subhalos. Despite the fact that the virial masses at infall are similar in the SPH and DM-only runs, the inner DM orbits have been expanded in the SPH runs (but, prior to infall, are not removed from the halo). As this paper has shown, the DM mass in the inner regions is lowered for those satellites with $\mathrm{M}_{*}>10^{7} M_{\odot}$ due to the effects of feedback prior to infall. After infall, SPH satellites are also prone to significantly more tidal stripping than their DM-only counterparts. Figure 8 summarizes our results on the combined impact of DM core-creation and tidal stripping on the internal dynamics of satellites. This figure shows the difference in $v_{c}$ at $1 \mathrm{kpc}$ at $z=0$ between SPH and DM-only satellites, as a function of $v_{\max }$ at infall of the DM-only satellites. The top panel of this Figure shows the change in the DM contribution to $v_{c}$ (again, the DM-only masses have been reduced by $f_{\text {bar }}$ for a direct comparison), while the bottom panel shows the change in the total $v_{c}$ at $1 \mathrm{kpc}$. We note that we exclude from the plot one data point that has a change in total $v_{c} \sim 25 \mathrm{~km} / \mathrm{s}$. This dramatic change is due to the fact that the SPH satellite has recently passed directly through the disk of its parent galaxy, stripping it more substantially than its DM-only counterpart (since there is no disk in the DM-only run).

A well defined trend for the change in DM mass interior to $1 \mathrm{kpc}$ between SPH and DM-only runs can be seen in the top panel of Figure 8 In order to quantify the change in DM mass that baryonic processes combined with tidal stripping induce in the central regions of satellites compared to the DM-only case, we fit a linear regression to the data points in the range $20<v_{\max }<50 \mathrm{~km} / \mathrm{s}$. The resulting fit, shown in the top panel of Figure 8 as the dashed line, is $\Delta\left(v_{c}, 1 k p c\right)=0.2 v_{\max , D M-o n l y}-0.26$ $\mathrm{km} / \mathrm{s}$.

The bottom panel of Figure 8 shows that the change in total $v_{c}$ at $1 \mathrm{kpc}$ between $\mathrm{SPH}$ and DM-only runs is slightly lower for gaseous satellites (open circles) than those satellites that are gas-free. Like the bottom panel of Figure 3. this is because baryons contribute significantly to the mass in the interior $1 \mathrm{kpc}$. While there is not much trend at infall visible in the bottom panel of Figure 3, a trend is beginning to emerge at $z=0$ in the bottom panel of Figure 8 . That is, as gas is removed from these systems after infall, the underlying difference in the DM central masses in the SPH and DM-only runs emerges. Note that it is likely that the gas-rich satellites in this work are artificially gas rich due to inefficient stripping of gas in SPH simulations. The trend in the bottom panel of Figure 8, therefore, would be even stronger if SPH did not suffer from this numerical effect.

We advocate that DM-only results apply the above linear relation to those halos that are accreted onto Milky Way-mass halos with $20<v_{\max }<50 \mathrm{~km} / \mathrm{s}$. Failure to account for these effects will over predict the central mass of luminous satellites such as those around the Milky Way and M31. This is likely the source of the

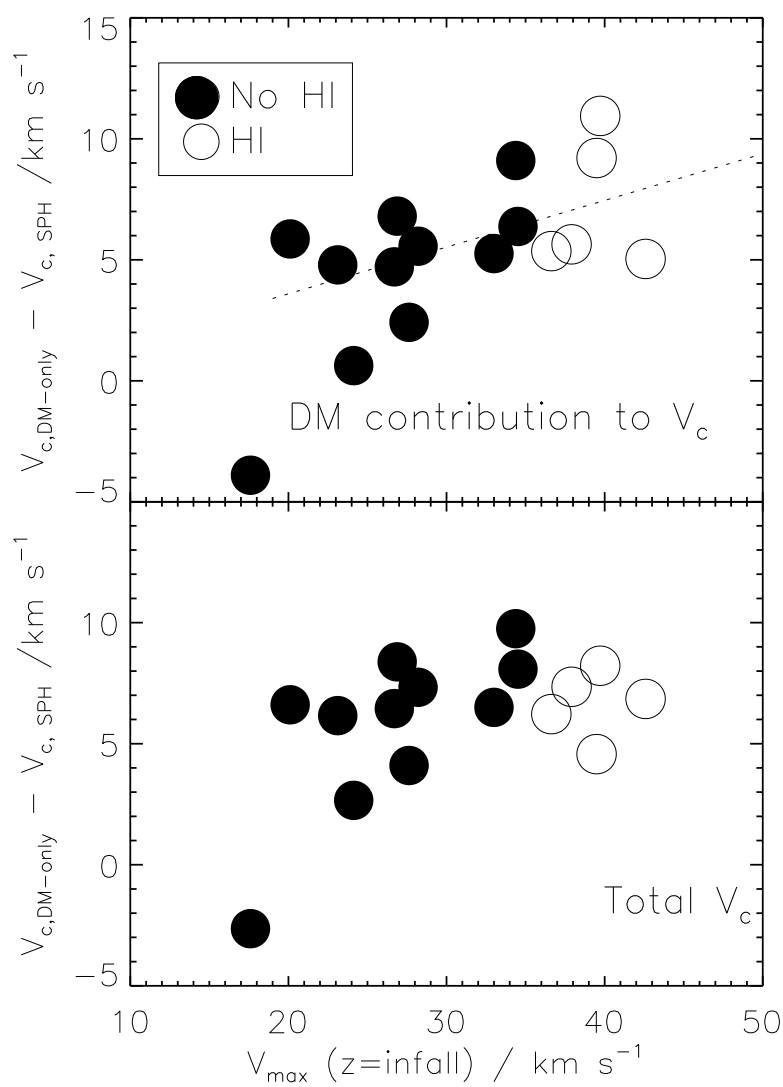

FIG. 8. - The difference in $v_{c}$ at $1 \mathrm{kpc}$ at $z=0$ between the SPH and DM-only counterparts, as a function of $V_{\max }$ of the DM-only satellite at infall. Top panel: The difference in the DM contribution to $v_{c}$ at $1 \mathrm{kpc}$ for matched $\mathrm{SPH}$ and DM-only subhalos. Bottom panel: The difference in total $v_{c}$ at $1 \mathrm{kpc}$.

tension discussed in Bovlan-Kolchin et al. (2011, 2012) between the densities in the MW's classical dSph population and the DM-only Aquarius subhalos. Those studies emphasized that the observed MW dSphs appear to be less dense than those found in a CDM DM-only simulation. Including the affects of baryonic physics reduces, and may even completely alleviate, this discrepancy. We explore the observational consequences of our updated model further in a companion paper (Brooks \& Zolotov 2012).

\section{CONCLUSION \& DISCUSSION}

We have demonstrated that the inclusion of baryonic physics can dramatically alter the evolution of bright satellite galaxies around Milky Way-massed galaxies. The population of satellites studied here have $V$-band magnitudes consistent with the range of the Milky Way's classical dSphs, $-15<M_{V}<-8$. Our sample contains both gas-free dSph analogs and massive gas-rich dwarfs. By directly comparing the internal properties of satellites simulated with gas hydrodynamics (SPH) to the same satellites in DM-only simulations, we have demonstrated the impact of baryons across a range of masses on satellite evolution. Our main results are summarized below.

- Before infall, the progenitors of luminous satellites $\left(M_{v i r}>10^{9} M_{\odot}, M_{\star}>10^{7} M_{\odot}\right)$ undergo rapid and frequent bursts of star formation. The associated 
SNe feedback from these SF episodes results in structural changes to the central mass distribution of these dwarf galaxies, resulting in reduced DM densities and shallower inner DM density profiles than DM-only galaxies.

- The progenitors of lower luminosity satellites $\left(M_{\star} \leq 10^{7} M_{\odot}\right.$ at infall) reduce their gas content, and hence become less efficient at forming stars, earlier than more massive dwarfs. This gas loss is partly due to heating by the uniform UV background, as well as subsequent gas loss in early star forming/feedback events, preventing them from having multiple strong bursts of star formation that lead to DM core creation. Low luminosity satellites, therefore, tend to retain steep DM density profiles that are comparable to DMonly runs.

- For SPH satellites across all masses, the overall reduction prior to infall in total $v_{c}$ is, on average, less than $5 \mathrm{~km} / \mathrm{s}$. Although SNe-driven outflows have reduced the central DM mass in halos with $M_{v i r}>10^{9} M_{\odot}$, the presence of gas keeps the overall central mass comparable to the DM-only case. However, this gas is stripped after infall. Hence, the major reduction in central mass is set in place within the DM component prior to infall, but the removal of gas is necessary to reduce the total mass between the SPH and DM-only runs by $z=0$.

- Once accreted, SPH satellites experience more mass loss due to tidal stripping than DM-only satellites, the amount of which is dependent on infall time and orbit. While both SPH and DM-only satellites are affected by tidal stripping, the presence of a baryonic disk in the SPH runs results in a greater reduction in the central $v_{c}$ in $\mathrm{SPH}$ satellites. The influence of a baryonic disk is especially strong for satellites with shallow DM density profiles. We find that SPH satellites with $z_{\text {infall }}>1$ experience a reduction in their central circular velocities that is $11-62 \%$ more than the reduction in $v_{c}$ at $1 \mathrm{kpc}$ experienced by their DM-only counterparts.

- By $z=0$, the combined effects of DM core creation and enhanced tidal stripping for luminous satellites results in a significant discrepancy between the circular velocity profiles of SPH and DM-only satellites. We find that the $v_{c}$ at $1 \mathrm{kpc}$ predicted for satellites by DM-only simulations should be reduced by $\Delta\left(v_{c}, 1 k p c\right) \sim 0.2 v_{\max , D M-o n l y}-0.26$ $\mathrm{km} / \mathrm{s}$, for satellites with $20<v_{\max }<50 \mathrm{~km} / \mathrm{s}$ at infall.

High resolution that allows simulators to limit star formation to high density peaks is an essential requirement to reproduce the baryonic effects in this paper. However, Governato et al. (2010) and Guedes et al. (2011) showed that, even at high resolution, if star formation is allowed to occur diffusely across the disk, no large scale outflows are generated. Restricting star formation to high density peaks instead leads to overpressurized regions of hot gas when stars go SNe, leading to outflows. These overpressurized regions expand faster than the local dynamical time. When this occurs in the central $\sim 1 \mathrm{kpc}$, the potential flattens as this hot gas expands, leading to an irreversible expansion of the DM orbits (Pontzen \& Governato 2012). Restricting star formation to high density peaks is comparable to allowing stars to only form in giant molecular clouds, rather than across the entire disk at any given time. The simulations used in this work are the first at these high resolutions to include metal line cooling (Shen et al. 2010) and a presciption for self-shielding of cold gas, allowing star formation to be tied to the shielded regions where $\mathrm{H}_{2}$ can form (Christensen et al. 2012).

It is important to note that simulations that do not resolve the effect of feedback at high densities will be unable to reproduce the results of this paper. However, the feedback model employed in this paper has been shown to match the observed mass - metallicity relation for galaxies as a function of redshift (Brooks et al. 2007; Maiolino et al. 2008), the baryonic Tully-Fisher relationship (Christensen et al. in prep.), the size - luminosity relation of galaxy disks (Brooks et al. 2011), the $z=0$ stellar mass to halo mass relation (Munshi et al. 2012), and the central mass as a function of stellar mass for galaxies in the luminosity range in this paper Governato et al. 2012). This large number of successes in matching the observed scaling relations of galaxies lends credence to the particular feedback model employed in this paper to study satellite galaxies.

In addition to reproducing the above scaling relations, the star formation and feedback model used in this work has been used to simulate bulgeless dwarf disk galaxies (Governato et al. 2010). Importantly, the processes that lead to bulgeless galaxies also transform cuspy DM density profiles into cored profiles, leading these simulations to match the central dark matter densities derived by the THINGS and LiTTLE THINGS surveys Oh et al. 2011; Governato et al. 2012). In other words, the simulations used in this work have been shown to reconcile the cusp/core problem in CDM. In this paper, we have shown that this same model can alleviate the tension between the dense, massive satellites predicted by CDM with the observations of lower density, luminous dSph satellites Bovlan-Kolchin et al. 2012; Wolf \& Bullock 2012; Hayashi \& Chiba 2012). We note that the processes described in this paper also act to reduce the overall number of massive subhalos that exist at $z=0$, potentially solving the missing satellites problem in CDM. Hence, it remains possible to resolve the small scale problems of CDM with a proper model for baryonic physics, and without invoking exotic forms of DM.

The results presented here show that using DM-only CDM simulations to study the internal dynamics of luminous satellites will lead to erroneous results. While it is safe to assign the most luminous satellites to the originally most massive halos, those massive halos will experience evolution that CDM DM-only runs don't account for. In Brooks \& Zolotov (2012), we address how the model presented here affects the interpretation of kinematic observations of the Milky Way's dSph population.

We thank the anonymous referee for comments that helped to improve this paper. We thank James Bullock, Michael Boylan-Kolchin, Jay Gallagher, Kim Venn, Ryan 
Leaman, and Alan McConnachie for useful discussions. Resources supporting this work were provided by the NASA High-End Computing (HEC) Program through the NASA Advanced Supercomputing (NAS) Division at Ames Research Center. This research was supported in part by the National Science Foundation under Grant No. NSF PHY11-25915. AZ acknowledges support from the Lady Davis Foundation. AB acknowledges sup- port from The Grainger Foundation. The work of AZ and AD has been partly supported by the ISF through grant 6/08, by GIF through grant G-1052-104.7/2009, by the DFG via German-Israeli project cooperation grant STE1869/1-1.GE625/15-1, and by an NSF grant AST1010033 at UCSC. BW acknowledges support from NSF grant AST-0908193. CC, TQ and FG were partly supported by NSF grant AST-0908499.

\section{REFERENCES}

Agertz, O., Moore, B., Stadel, J., Potter, D., Miniati, F., Read, J., Mayer, L., Gawryszczak, A., Kravtsov, A., Nordlund, A., Pearce, F., Quilis, V., Rudd, D., Springel, V., Stone, J., Tasker E., Teyssier, R., Wadsley, J., \& Walder, R. 2007, MNRAS, 380, 963

Alam, S. M. K., Bullock, J. S., \& Weinberg, D. H. 2002, ApJ, 572,34

Barnes, J., \& Efstathiou, G. 1987, ApJ, 319, 575

Behroozi, P. S., Conroy, C., \& Wechsler, R. H. 2010, ApJ, 717, 379

Benson, A. J., Frenk, C. S., Lacey, C. G., Baugh, C. M., \& Cole, S. 2002, MNRAS, 333, 177

Bigiel, F., Leroy, A., Walter, F., Brinks, E., de Blok, W. J. G., Madore, B., \& Thornley, M. D. 2008, AJ, 136

Bigiel, F., Walter, F., Blitz, L., Brinks, E., de Blok, W. J. G., \& Madore, B. F. 2010, AJ, 140, 1194

Blanc, G. A., Heiderman, A., Gebhardt, K., Evans, N. J., \& Adams, J. 2009, ApJ, 704, 842

Bovill, M. S., \& Ricotti, M. 2011, ApJ, 741, 17

Boylan-Kolchin, M., Bullock, J. S., \& Kaplinghat, M. 2011, MNRAS, 415, L40

-. 2012, MNRAS, 2657

Boylan-Kolchin, M., \& Ma, C.-P. 2007, MNRAS, 374, 1227

Brook, C. B., Governato, F., Roškar, R., Stinson, G., Brooks, A. M., Wadsley, J., Quinn, T., Gibson, B. K., Snaith, O., Pilkington, K., House, E., \& Pontzen, A. 2011, MNRAS, 415, 1051

Brooks, A. M., Governato, F., Booth, C. M., Willman, B., Gardner, J. P., Wadsley, J., Stinson, G., \& Quinn, T. 2007, ApJ, 655, L17

Brooks, A. M., Solomon, A. R., Governato, F., McCleary, J., MacArthur, L. A., Brook, C. B. A., Jonsson, P., Quinn, T. R., \& Wadsley, J. 2011, ApJ, 728, 51

Brooks, A. M., \& Zolotov, A. 2012, ArXiv:1207.2468

Bullock, J. S., Kravtsov, A. V., \& Weinberg, D. H. 2000, ApJ, 539,517

Busha, M. T., Alvarez, M. A., Wechsler, R. H., Abel, T., \& Strigari, L. E. 2010, ApJ, 710, 408

Ceverino, D., \& Klypin, A. 2009, ApJ, 695, 292

Choi, J.-H., Weinberg, M. D., \& Katz, N. 2009, MNRAS, 400, 1247

Christensen, C., Quinn, T., Governato, F., Stilp, A., Shen, S., \& Wadsley, J. 2012, ArXiv e-prints

Christensen, C. R., Quinn, T., Stinson, G., Bellovary, J., \& Wadsley, J. 2010, ApJ, 717, 121

Cloet-Osselaer, A., De Rijcke, S., Schroyen, J., \& Dury, V. 2012, MNRAS, 2952

Colín, P., Avila-Reese, V., Vázquez-Semadeni, E., Valenzuela, O., \& Ceverino, D. 2010, ApJ, 713, 535

Conroy, C., \& Wechsler, R. H. 2009, ApJ, 696, 620

de Blok, W. J. G., \& Bosma, A. 2002, A\&A, 385, 816

de Blok, W. J. G., McGaugh, S. S., \& Rubin, V. C. 2001, AJ, 122,2396

de Blok, W. J. G., Walter, F., Brinks, E., Trachternach, C., Oh, S., \& Kennicutt, R. C. 2008, AJ, 136, 2648

de Souza, R. S., Rodrigues, L. F. S., Ishida, E. E. O., \& Opher, R. 2011, MNRAS, 415, 2969

Dekel, A., Arad, I., Devor, J., \& Birnboim, Y. 2003a, ApJ, 588, 680

Dekel, A., Devor, J., \& Hetzroni, G. 2003b, MNRAS, 341, 326

Dekel, A., \& Silk, J. 1986, ApJ, 303, 39

Dekel, A., \& Woo, J. 2003, MNRAS, 344, 1131

Del Popolo, A. 2012, MNRAS, 419, 971
Di Cintio, A., Knebe, A., Libeskind, N. I., Brook, C., Yepes, G., Gottloeber, S., \& Hoffman, Y. 2012, ArXiv e-prints

Donato, F., Gentile, G., Salucci, P., Frigerio Martins, C., Wilkinson, M. I., Gilmore, G., Grebel, E. K., Koch, A., \& Wyse, R. 2009, MNRAS, 397, 1169

D'Onghia, E., Springel, V., Hernquist, L., \& Keres, D. 2010, ApJ, 709,1138

Efstathiou, G., Bond, J. R., \& White, S. D. M. 1992, MNRAS, 258, 1P

Feldmann, R., Gnedin, N. Y., \& Kravtsov, A. V. 2011, ApJ, 732, 115

Geen, S., Slyz, A., \& Devriendt, J. 2012, ArXiv e-prints

Gentile, G., Salucci, P., Klein, U., \& Granato, G. L. 2007, MNRAS, 375, 199

Gill, S. P. D., Knebe, A., \& Gibson, B. K. 2004, MNRAS, 351, 399

Gilmore, G., Wilkinson, M., Kleyna, J., Koch, A., Evans, W.

Wyse, R. F. G., \& Grebel, E. K. 2007, Nuclear Physics B Proceedings Supplements, 173, 15

Gnedin, N. Y. 2000, ApJ, 542, 535

Gnedin, N. Y., \& Kravtsov, A. V. 2006, ApJ, 645, 1054

—. 2010, ApJ, 714, 287

Gnedin, N. Y., Tassis, K., \& Kravtsov, A. V. 2009, ApJ, 697, 55

Goerdt, T., Moore, B., Read, J. I., Stadel, J., \& Zemp, M. 2006, MNRAS, 368, 1073

Governato, F., Brook, C., Mayer, L., Brooks, A., Rhee, G., Wadsley, J., Jonsson, P., Willman, B., Stinson, G., Quinn, T., \& Madau, P. 2010, Nature, 463, 203

Governato, F., Brook, C. B., Brooks, A. M., Mayer, L., Willman, B., Jonsson, P., Stilp, A. M., Pope, L., Christensen, C., Wadsley, J., \& Quinn, T. 2009, MNRAS, 398, 312

Governato, F., Willman, B., Mayer, L., Brooks, A., Stinson, G., Valenzuela, O., Wadsley, J., \& Quinn, T. 2007, MNRAS, 374, 1479

Governato, F., Zolotov, A., Pontzen, A., Christensen, C., Oh, S. H., Brooks, A. M., Quinn, T., Shen, S., \& Wadsley, J. 2012, MNRAS, 422, 1231

Gross, M. A. K. 1997, PhD thesis, UNIVERSITY OF CALIFORNIA, SANTA CRUZ

Guedes, J., Callegari, S., Madau, P., \& Mayer, L. 2011, ApJ, 742, 76

Guo, Q., White, S., Li, C., \& Boylan-Kolchin, M. 2010, MNRAS, 404,1111

Haardt, F., \& Madau, P. 2001, in Clusters of Galaxies and the High Redshift Universe Observed in X-rays, ed. D. M. Neumann \& J. T. V. Tran

Hayashi, E., Navarro, J. F., Taylor, J. E., Stadel, J., \& Quinn, T. 2003, ApJ, 584, 541

Hayashi, K., \& Chiba, M. 2012, ArXiv e-prints

Hoeft, M., Yepes, G., Gottlöber, S., \& Springel, V. 2006, MNRAS, 371, 401

Jardel, J. R., \& Gebhardt, K. 2012, ApJ, 746, 89

Katz, N., \& White, S. D. M. 1993, ApJ, 412, 455

Kazantzidis, S., Łokas, E. L., Callegari, S., Mayer, L., \& Moustakas, L. A. 2011, ApJ, 726, 98

Kazantzidis, S., Mayer, L., Mastropietro, C., Diemand, J., Stadel, J., \& Moore, B. 2004, ApJ, 608, 663

Kleyna, J., Wilkinson, M. I., Evans, N. W., Gilmore, G., \& Frayn, C. 2002, MNRAS, 330, 792

Kleyna, J. T., Wilkinson, M. I., Gilmore, G., \& Evans, N. W. 2003, ApJ, 588, L21

Klimentowski, J., Łokas, E. L., Knebe, A., Gottlöber, S., Martinez-Vaquero, L. A., Yepes, G., \& Hoffman, Y. 2010, MNRAS, 402, 1899 
Klypin, A., Kravtsov, A. V., Valenzuela, O., \& Prada, F. 1999, ApJ, 522, 82

Klypin, A. A., Trujillo-Gomez, S., \& Primack, J. 2011, ApJ, 740, 102

Knollmann, S. R., \& Knebe, A. 2009, ApJS, 182, 608

Koposov, S. E., Yoo, J., Rix, H.-W., Weinberg, D. H., Macciò, A. V., \& Escudé, J. M. 2009, ApJ, 696, 2179

Kravtsov, A. V., Gnedin, O. Y., \& Klypin, A. A. 2004, ApJ, 609, 482

Kroupa, P. 2001, MNRAS, 322, 231

Kroupa, P., Tout, C. A., \& Gilmore, G. 1993, MNRAS, 262, 545

Krumholz, M. R., \& Dekel, A. 2011, ArXiv e-prints

Kuhlen, M., Krumholz, M. R., Madau, P., Smith, B. D., \& Wise, J. 2012, ApJ, 749, 36

Kuzio de Naray, R., McGaugh, S. S., de Blok, W. J. G., \& Bosma, A. 2006, ApJS, 165, 461

Leauthaud, A., George, M. R., Behroozi, P. S., Bundy, K. Tinker, J., Wechsler, R. H., Conroy, C., Finoguenov, A., \& Tanaka, M. 2012, ApJ, 746, 95

Leitherer, C., Schaerer, D., Goldader, J. D., Delgado, R. M. G., Robert, C., Kune, D. F., de Mello, D. F., Devost, D., \& Heckman, T. M. 1999, ApJS, 123, 3

Leitner, S. N. 2012, ApJ, 745, 149

Leroy, A. K., Walter, F., Brinks, E., Bigiel, F., de Blok, W. J. G., Madore, B. F., \& Thornley, M. D. 2008, AJ, 136, 2782

Lia, C., Carraro, G., \& Salucci, P. 2000, A\&A, 360, 76

Lovell, M. R., Eke, V., Frenk, C. S., Gao, L., Jenkins, A., Theuns, T., Wang, J., White, S. D. M., Boyarsky, A., \& Ruchayskiy, O. 2012, MNRAS, 420, 2318

Macciò, A. V., Kang, X., \& Moore, B. 2009, ApJ, 692, L109

Macciò, A. V., Stinson, G., Brook, C. B., Wadsley, J., Couchman, H. M. P., Shen, S., Gibson, B. K., \& Quinn, T. 2012, ApJ, 744, L9

Madau, P., Diemand, J., \& Kuhlen, M. 2008, ApJ, 679, 1260

Maiolino, R., Nagao, T., Grazian, A., Cocchia, F., Marconi, A., Mannucci, F., Cimatti, A., Pipino, A., Ballero, S., Calura, F., Chiappini, C., Fontana, A., Granato, G. L., Matteucci, F. Pastorini, G., Pentericci, L., Risaliti, G., Salvati, M., \& Silva, L. 2008, A\&A, 488, 463

Maller, A. H., \& Dekel, A. 2002, MNRAS, 335, 487

Mashchenko, S., Couchman, H. M. P., \& Sills, A. 2005, ApJ, 624, 726

Mashchenko, S., Couchman, H. M. P., \& Wadsley, J. 2006, Nature, 442, 539

Mashchenko, S., Wadsley, J., \& Couchman, H. M. P. 2008, Science, 319, 174

Mayer, L., Governato, F., Colpi, M., Moore, B., Quinn, T., Wadsley, J., Stadel, J., \& Lake, G. 2001, ApJ, 559, 754

Mayer, L., Mastropietro, C., Wadsley, J., Stadel, J., \& Moore, B. 2006, MNRAS, 369, 1021

McConnachie, A. W. 2012, AJ, 144, 4

Mitchell, N. L., McCarthy, I. G., Bower, R. G., Theuns, T., \& Crain, R. A. 2009, MNRAS, 395, 180

Moore, B., Ghigna, S., Governato, F., Lake, G., Quinn, T., Stadel, J., \& Tozzi, P. 1999, ApJ, 524, L19

Moster, B. P., Somerville, R. S., Maulbetsch, C., van den Bosch, F. C., Macciò, A. V., Naab, T., \& Oser, L. 2010, ApJ, 710, 903

Munshi, F., Governato, F., Brooks, A. M., Christensen, C., Shen, S., Loebman, S., Moster, B., Quinn, T., \& Wadsley, J. 2012, ArXiv e-prints

Navarro, J. F., Eke, V. R., \& Frenk, C. S. 1996, MNRAS, 283, L72

Navarro, J. F., Frenk, C. S., \& White, S. D. M. 1997, ApJ, 490, 493

Navarro, J. F., Ludlow, A., Springel, V., Wang, J., Vogelsberger, M., White, S. D. M., Jenkins, A., Frenk, C. S., \& Helmi, A. 2010, MNRAS, 402, 21

Navarro, J. F., \& Steinmetz, M. 1997, ApJ, 478, 13

Neistein, E., Weinmann, S. M., Li, C., \& Boylan-Kolchin, M. 2011, MNRAS, 414, 1405

Nickerson, S., Stinson, G., Couchman, H. M. P., Bailin, J., \& Wadsley, J. 2011, MNRAS, 415, 257

Ogiya, G., \& Mori, M. 2012, ArXiv e-prints

Oh, S.-H., Brook, C., Governato, F., Brinks, E., Mayer, L., de Blok, W. J. G., Brooks, A., \& Walter, F. 2011, AJ, 142, 24

Okamoto, T., Gao, L., \& Theuns, T. 2008, MNRAS, 390, 920

Ostriker, J. P., \& McKee, C. F. 1988, Reviews of Modern Physics, 60,1
Parry, O. H., Eke, V. R., Frenk, C. S., \& Okamoto, T. 2012, MNRAS, 419, 3304

Pasetto, S., Grebel, E. K., Berczik, P., Spurzem, R., \& Dehnen, W. 2010, A\&A, 514, A47

Peñarrubia, J., Benson, A. J., Walker, M. G., Gilmore, G., McConnachie, A. W., \& Mayer, L. 2010, MNRAS, 406, 1290

Peñarrubia, J., McConnachie, A. W., \& Navarro, J. F. 2008, ApJ, 672,904

Peebles, P. J. E. 1969, ApJ, 155, 393

Persic, M., Salucci, P., \& Stel, F. 1996, MNRAS, 281, 27

Pontzen, A., \& Governato, F. 2012, MNRAS, 421, 3464

Power, C., Navarro, J. F., Jenkins, A., Frenk, C. S., White,

S. D. M., Springel, V., Stadel, J., \& Quinn, T. 2003, MNRAS, 338,14

Quinn, T., Katz, N., \& Efstathiou, G. 1996, MNRAS, 278, L49

Raiteri, C. M., Villata, M., \& Navarro, J. F. 1996, A\&A, 315, 105

Read, J. I., \& Gilmore, G. 2005, MNRAS, 356, 107

Read, J. I., Wilkinson, M. I., Evans, N. W., Gilmore, G., \&

Kleyna, J. T. 2006a, MNRAS, 367, 387

-. 2006b, MNRAS, 366, 429

Reed, D., Governato, F., Quinn, T., Gardner, J., Stadel, J., \& Lake, G. 2005, MNRAS, 359, 1537

Riess, A. G., Filippenko, A. V., Challis, P., Clocchiatti, A., Diercks, A., Garnavich, P. M., Gilliland, R. L., Hogan, C. J., Jha, S., Kirshner, R. P., Leibundgut, B., Phillips, M. M., Reiss, D., Schmidt, B. P., Schommer, R. A., Smith, R. C., Spyromilio, J., Stubbs, C., Suntzeff, N. B., \& Tonry, J. 1998, AJ, 116, 1009

Robertson, B. E., \& Kravtsov, A. V. 2008, ApJ, 680, 1083

Romano-Díaz, E., Shlosman, I., Heller, C., \& Hoffman, Y. 2010, ApJ, 716, 1095

Saitoh, T. R., Daisaka, H., Kokubo, E., Makino, J., Okamoto, T., Tomisaka, K., Wada, K., \& Yoshida, N. 2008, PASJ, 60, 667

Salucci, P., Lapi, A., Tonini, C., Gentile, G., Yegorova, I., \& Klein, U. 2007, MNRAS, 378, 41

Salucci, P., Wilkinson, M. I., Walker, M. G., Gilmore, G. F., Grebel, E. K., Koch, A., Frigerio Martins, C., \& Wyse, R. F. G. 2012, MNRAS, 420, 2034

Sawala, T., Frenk, C. S., Crain, R. A., Jenkins, A., Schaye, J., Theuns, T., \& Zavala, J. 2012, ArXiv e-prints

Sawala, T., Guo, Q., Scannapieco, C., Jenkins, A., \& White, S. 2011, MNRAS, 413, 659

Sawala, T., Scannapieco, C., Maio, U., \& White, S. 2010, MNRAS, 402, 1599

Schruba, A., Leroy, A. K., Walter, F., Bigiel, F., Brinks, E., de Blok, W. J. G., Dumas, G., Kramer, C., Rosolowsky, E., Sandstrom, K., Schuster, K., Usero, A., Weiss, A., \& Wiesemeyer, H. 2011, A.J, 142, 37

Shen, S., Wadsley, J., \& Stinson, G. 2010, MNRAS, 1043

Simha, V., Weinberg, D. H., Davé, R., Fardal, M., Katz, N., \& Oppenheimer, B. D. 2012, MNRAS, 3117

Simon, J. D., Bolatto, A. D., Leroy, A., \& Blitz, L. 2003, ApJ, 596,957

Simon, J. D., \& Geha, M. 2007, ApJ, 670, 313

Spano, M., Marcelin, M., Amram, P., Carignan, C., Epinat, B., \& Hernandez, O. 2008, MNRAS, 383, 297

Spergel, D. N., Bean, R., Doré, O., Nolta, M. R., Bennett, C. L. Dunkley, J., Hinshaw, G., Jarosik, N., Komatsu, E., Page, L., Peiris, H. V., Verde, L., Halpern, M., Hill, R. S., Kogut, A., Limon, M., Meyer, S. S., Odegard, N., Tucker, G. S., Weiland, J. L., Wollack, E., \& Wright, E. L. 2007, ApJS, 170, 377

Springel, V., Wang, J., Vogelsberger, M., Ludlow, A., Jenkins, A., Helmi, A., Navarro, J. F., Frenk, C. S., \& White, S. D. M. 2008, MNRAS, 391, 1685

Stinson, G., Seth, A., Katz, N., Wadsley, J., Governato, F., \& Quinn, T. 2006, MNRAS, 373, 1074

Stoehr, F., White, S. D. M., Tormen, G., \& Springel, V. 2002, MNRAS, 335, L84

Strigari, L. E., Bullock, J. S., Kaplinghat, M., Diemand, J., Kuhlen, M., \& Madau, P. 2007a, ApJ, 669, 676

Strigari, L. E., Bullock, J. S., Kaplinghat, M., Kravtsov, A. V., Gnedin, O. Y., Abazajian, K., \& Klypin, A. A. 2006, ApJ, 652, 306

Strigari, L. E., Frenk, C. S., \& White, S. D. M. 2010, MNRAS, 408,2364

Strigari, L. E., Kaplinghat, M., \& Bullock, J. S. 2007b,

Phys. Rev. D, 75, 061303

Susa, H. 2008, ApJ, 684, 226 
Swaters, R. A., Madore, B. F., van den Bosch, F. C., \& Balcells, M. 2003, ApJ, 583, 732

Tasker, E. J., \& Bryan, G. L. 2008, ApJ, 673, 810

Taylor, J. E., \& Babul, A. 2001, ApJ, 559, 716

Teyssier, R., Pontzen, A., Dubois, Y., \& Read, J. 2012, ArXiv e-prints

Thielemann, F., Nomoto, K., \& Yokoi, K. 1986, A\&A, 158, 17

Thoul, A. A., \& Weinberg, D. H. 1996, ApJ, 465, 608

Tollerud, E. J., Bullock, J. S., Strigari, L. E., \& Willman, B. 2008, ApJ, 688, 277

Trachternach, C., de Blok, W. J. G., Walter, F., Brinks, E., \& Kennicutt, Jr., R. C. 2008, AJ, 136, 2720

van den Aarssen, L. G., Bringmann, T., \& Pfrommer, C. 2012, ArXiv e-prints

van den Bosch, F. C., Robertson, B. E., Dalcanton, J. J., \& de Blok, W. J. G. 2000, AJ, 119, 1579

Vázquez, G. A., \& Leitherer, C. 2005, ApJ, 621, 695

Vera-Ciro, C. A., Helmi, A., Starkenburg, E., \& Breddels, M. A. 2012, ArXiv e-prints

Vogelsberger, M., Zavala, J., \& Loeb, A. 2012, ArXiv e-prints

Wadepuhl, M., \& Springel, V. 2011, MNRAS, 410, 1975

Wadsley, J. W., Stadel, J., \& Quinn, T. 2004, New Astronomy, 9, 137
Walker, M. G., Mateo, M., Olszewski, E. W., Peñarrubia, J.,

Wyn Evans, N., \& Gilmore, G. 2009, ApJ, 704, 1274

Walker, M. G., \& Peñarrubia, J. 2011, ApJ, 742, 20

Walsh, S. M., Willman, B., \& Jerjen, H. 2009, AJ, 137, 450

Wang, J., Frenk, C. S., Navarro, J. F., \& Gao, L. 2012, ArXiv e-prints

Weisz, D. R., Dalcanton, J. J., Williams, B. F., Gilbert, K. M. Skillman, E. D., Seth, A. C., Dolphin, A. E., McQuinn, K. B. W., Gogarten, S. M., Holtzman, J., Rosema, K., Cole, A., Karachentsev, I. D., \& Zaritsky, D. 2011, ApJ, 739, 5

Weldrake, D. T. F., de Blok, W. J. G., \& Walter, F. 2003, MNRAS, 340, 12

Wetzel, A. R., \& White, M. 2010, MNRAS, 403, 1072

Wolf, J., \& Bullock, J. S. 2012, ArXiv e-prints

Woosley, S. E., \& Weaver, T. A. 1995, ApJS, 101, 181

Yang, X., Mo, H. J., van den Bosch, F. C., Zhang, Y., \& Han, J. 2011, ArXiv e-prints

Zentner, A. R., \& Bullock, J. S. 2003, ApJ, 598, 49

Zolotov, A., Willman, B., Brooks, A. M., Governato, F., Brook, C. B., Hogg, D. W., Quinn, T., \& Stinson, G. 2009, ApJ, 702, 1058

APPENDIX

NUMERICAL CONVERGENCE

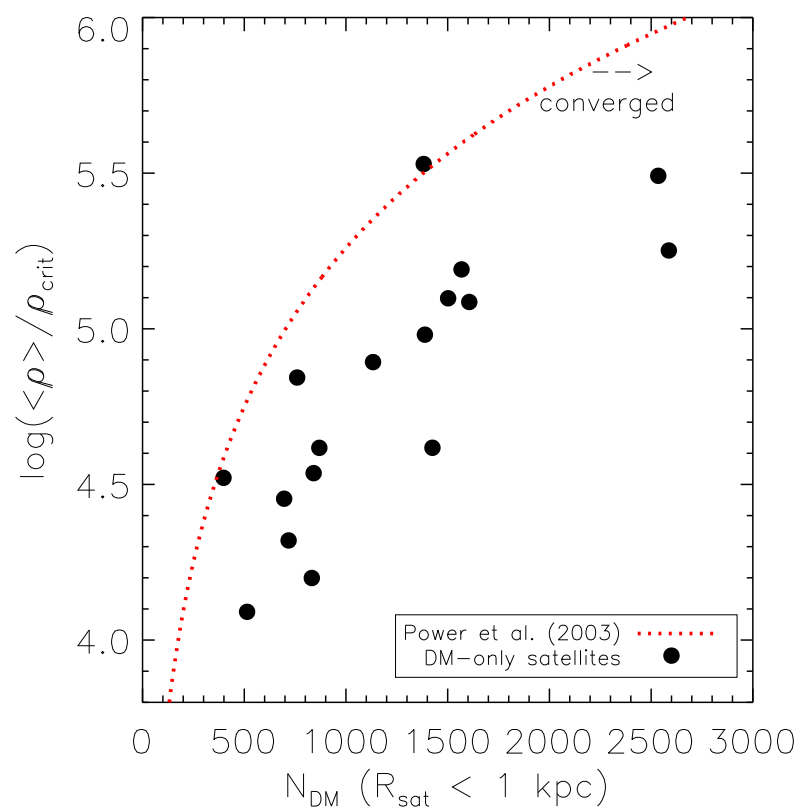

FIG. 9. - The mean enclosed density at $1 \mathrm{kpc}$, as a function of the number of DM particles within the same radius, for DM-only satellites at infall. The density profiles of all but one of these satellites has converged by 1 kpc, according to the Power et al. (2003) criteria. The density profile of the remaining satellite has converged by $1.1 \mathrm{kpc}$. According to this criteria, the density profiles of 15 out of the 17 satellites have already converged at $r=0.80 \mathrm{kpc}$

The central densities of simulated halos can be artificially low due to particle relaxation when a halo contains too few particles (e.g., Power et al. 2003). In this appendix, we demonstrate that the resolution of our simulations is sufficient to study the central density profiles of the satellites. We verify that the creation of shallow density cores prior to infall is the result of physical processes (SN-driven outflows) and not due to low resolution in the central regions of the satellites.

Power et al. (2003) have shown that the density profile of a simulated galaxy converges at a radius where the two-body relaxation time is larger than the Hubble time. The radius of convergence encloses enough particles to satisfy the following criteria:

$$
\frac{\sqrt{(200)}}{8} \frac{N(r)}{\ln N(r)}\left(\frac{\bar{\rho}}{\rho_{\text {crit }}}\right)^{-1 / 2}>0.6
$$


where $\mathrm{N}(\mathrm{r})$ is the number of particles enclosed at a given radius, and $\bar{\rho}$ is the mean enclosed density at that radius. As we showed in this paper, SPH satellites with $M_{*}>10^{7} M_{\odot}$ at infall develop lower central densities, and since the above criterion is dependent on enclosed density, they will meet this criterion with fewer particles than DM-only satellites with steep density profiles. We therefore apply this criterion to our DM-only runs, as they will require more particles at a given radius to be converged. We then require that properties of the SPH runs be measured at a radius where the DM-only runs converge.

The Power criteria has been tested and verified for host galaxies in DM-only simulations, but has not yet been validated for subhalos in simulations. We therefore test the convergence of our DM-only runs at infall, before these satellites undergo mass (and hence particle) loss due to tidal stripping, but after the time that DM cores have been created in the massive SPH runs. This allows us to test if the density profiles of the SPH satellites before infall are poorly resolved, which would lead to artificially shallow density profiles before infall. Figure 9 shows the mean enclosed density at $1 \mathrm{kpc}$, as a function of the number of DM particles within $1 \mathrm{kpc}$, for the 17 matched DM-only satellites at infall. All but one of our satellites has converged at this radius according to the Power criteria, and we have verified that the remaining DM-only satellite has converged by $1.1 \mathrm{kpc}$. In fact, the density profiles of 15 out of the 17 satellites have already converged at $r=0.80 \mathrm{kpc}$, according to this criteria.

At $\mathrm{z}=0$, the central density profiles of the DM-only satellites have slopes of $-2.2<\alpha(1 \mathrm{kpc})<-1.8$ across their mass range, consistent with the results of previous studies of DM-only halos (e.g., Reed et al. 2005). Springel et al. (2008) examined the convergence of density profiles in very high resolution DM-only simulations (the Aquarius simulations), and found the density converged at $4-6 \times$ the force softening length, $\epsilon$. We verified, using a lower resolution DM-only run with $1.5 \times$ larger force softening, that the density slopes in these simulations converge by $4 \epsilon$, or 700 pc. We also confirmed that $v_{c}$ is $90 \%$ converged by $1 \mathrm{kpc}$. The $v_{c}$ convergence radius is larger than for density, because $v_{c}$ is a cumulative quantity.

Additionally, we can test whether the density profiles of our SPH satellites are in agreement with higher resolution simulation studies of dwarf galaxies. As discussed throughout this paper, Governato et al. (2012, hereafter, G12) showed that SN-driven outflows create DM cores in isolated field dwarfs with $M_{*}>10^{7} M_{\odot}$. These isolated dwarf simulations were run with the same star formation and feedback scheme as the simulations used in this paper, but at $8 \times$ higher mass resolution and twice the force resolution. Two field dwarfs in the G12 sample have comparable stellar masses to the satellites studied here, one with $M_{*}=6 \times 10^{7} M_{\odot}$ and one with $M_{*}=3 \times 10^{6} M_{\odot}$. Figure 10 shows the DM density profiles of our most luminous satellites (which initially contain the highest number of particles and are therefore the best resolved, left panel) and our least luminous satellites (containing the fewest particles and should be least resolved, right panel) at $\mathrm{z}=0$. We have normalized the density of the galaxies in each panel at $1 \mathrm{kpc}$, such that all galaxies have the same $\rho(1 \mathrm{kpc})$, for a clear comparison of their DM density slopes. Two results are evident in Figure 10, First, the low-luminosity dwarfs in the right panel all have steep DM density profiles, while the more luminous dwarfs in the left panel have shallower DM profiles within $1 \mathrm{kpc}$. Second, the satellite density slopes in each mass range interior to $1 \mathrm{kpc}$ are comparable to the higher resolution G12 results (profiles at radii larger than $1 \mathrm{kpc}$ can be steeper in the satellites due to the tidal stripping they have experienced). We verified that the central density profiles of these two samples at infall also matches the $z=0$ G12 slopes, indicating little evolution in the central density profiles between infall and $z=0$. The comparable slopes of the satellite profiles to the more highly resolved field dwarfs is further evidence that the density profiles of our satellites have converged by $0.7 \mathrm{kpc}$. 

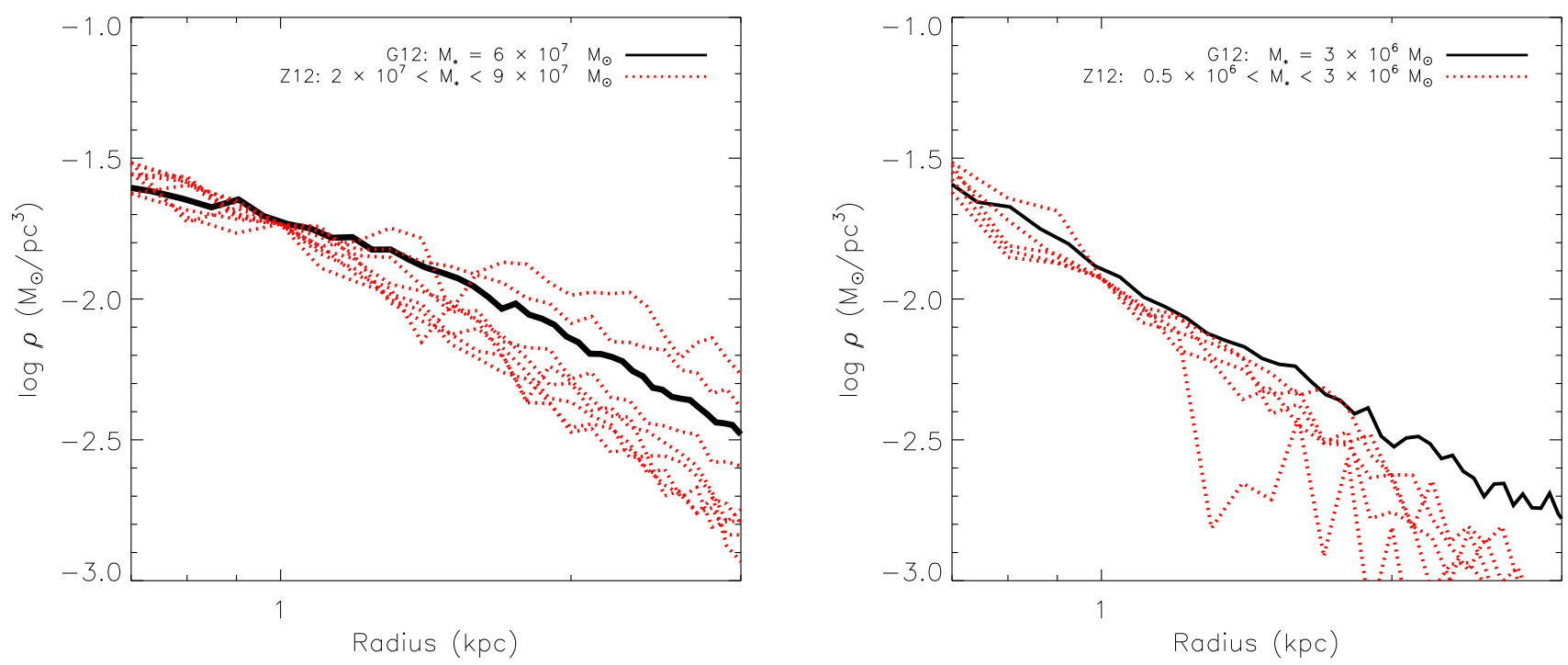

Fig. 10. - In this figure, we show the $z=0 \mathrm{DM}$ density profiles of satellites from this paper (in red dotted lines), as well as isolated field dwarfs from Governato et al. (2012, in black solid lines). Left Panel: The most luminous satellites in our sample; Right Panel: The least luminous galaxies in our sample. The legend lists the total stellar mass of these dwarf galaxies at $z=0$. 